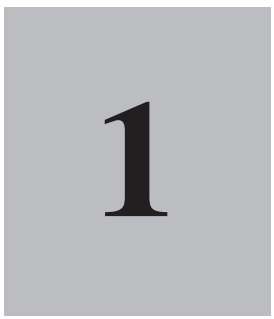

\title{
Fundamentals of Magnetic Devices
}

\subsection{Introduction}

Many electronic circuits require the use of inductors and transformers [1]-[47]. These are usually the largest, heaviest, and most expensive components in a circuit. They are defined by their electromagnetic behavior. The main feature of an inductor is its ability to store magnetic energy in the form of a magnetic field. The important feature of a transformer is its ability to couple magnetic fluxes of different windings and transfer ac energy from the input to the output through the magnetic field. The amount of energy transferred is determined by the operating frequency, flux density, and temperature. Transformers are used to change the ac voltage and current levels as well as to provide $\mathrm{dc}$ isolation while transmitting ac signals. They can combine energy from many ac sources by the addition of the magnetic flux and deliver the energy from all the inputs to one or multiple outputs simultaneously. The magnetic components are very important in power electronics and other areas of electrical engineering. Power losses in inductors and transformers are due to skin and proximity effects in windings, as well as to eddy currents and hysteresis in magnetic cores. Failure mechanisms in magnetic components are mostly due to excessive temperature rise. Therefore, these devices should satisfy both magnetic requirements and thermal limitations.

In this chapter, fundamental laws, quantities, and units of the magnetic theory are reviewed. Magnetic relationships are given and an equation for the inductance is derived. Hysteresis and eddy-current losses are studied. There are two kinds of eddy-current effects: skin effect and proximity effect. Both of these effects cause nonuniform distribution of the current density in conductors and increase the conductor ac resistance at high frequencies. The winding and core losses are determined. The winding resistance of magnetic components is studied using Dowell's equation [1]. Three shapes of winding conductors are considered: rectangular, square, and round. Properties of magnetic materials are also discussed. 


\subsection{Magnetic Relationships}

The magnetic field is characterized by magnetomotive force $\mathcal{F}$, magnetic field intensity $H$, magnetic flux density $B$, magnetic flux $\phi$, and magnetic flux linkage $\lambda$.

\subsubsection{Magnetomotive Force}

An inductor with $N$ turns carrying an ac current $i$ produces the magnetomotive force (MMF), which is also called the magnetomotance. The MMF is given by

$$
\mathcal{F}=N i(\mathrm{~A} \cdot \text { turns }) .
$$

Its descriptive unit is the ampere-turn (A $t$ ). However, the approved SI unit of the MMF is the ampere (A). The MMF is a source in magnetic circuits. The magnetic flux $\phi$ is forced to flow in a magnetic circuit by the MMF $\mathcal{F}=N i$ driving the magnetic circuit. Every time another complete turn with the current $i$ is added, the result of the integration increases by current $i$. The magnetomotive force is analogous to the electromotive force, which causes a current flow $i$.

\subsubsection{Magnetic Field Intensity}

The magnetic field intensity (or magnetic field strength) is given by

$$
H=\frac{\mathcal{F}}{l}=\frac{N i}{l}\left(\frac{\mathrm{A}}{\mathrm{m}}\right),
$$

where $l$ is the inductor length and $N$ is the number of turns.

\subsubsection{Magnetic Flux}

The amount of magnetic flux passing through a surface $S$ is given by

$$
\phi=\iint_{S} \mathbf{B} \cdot d \mathbf{S}(\mathrm{Wb}) .
$$

The unit of the magnetic flux is the weber. If the magnetic flux is uniform and perpendicular to the surface $A$, the amount of the magnetic flux passing through the surface $A$ is

$$
\phi=A B \quad(\mathrm{~Wb}) .
$$

The direction of a magnetic flux $\phi$ is determined by the right-hand rule. This rule states that if the fingers of the right hand encircle a coil in the direction of the current $i$, the thumb indicates the direction of the magnetic flux $\phi$.

\subsubsection{Magnetic Flux Density}

The magnetic flux density, or induction, is given by

$$
B=\frac{\phi}{A}(\mathrm{~T}) .
$$


The relationship between the magnetic flux density and the magnetic field intensity is given by

$$
B=\mu H=\mu_{r} \mu_{0} H=\frac{\mu N i}{l}=\frac{\mu \mathcal{F}}{l}(\mathrm{~T})
$$

where the permeability of free space is

$$
\mu_{0}=4 \pi \times 10^{-7}(\mathrm{H} / \mathrm{m}) ;
$$

$\mu=\mu_{r} \mu_{0}$ is the permeability, and $\mu_{r}=\mu / \mu_{0}$ is the relative permeability (i.e., relative to free space). For free space, insulators, and nonmagnetic conductors, $\mu_{r}=1$. For diamagnetics such as copper $(\mathrm{Cu})$, lead $(\mathrm{Pb})$, silver $(\mathrm{Ag})$, and gold $(\mathrm{Au}), \mu_{r} \approx 1-10^{-5} \approx 1$. However, for ferromagnetic materials such as iron (Fe), cobalt (Co), nickel (Ni), and their alloys, $\mu_{r}>1$ and it can be as high as 100000 . The permeability is the measure of the ability of a material to conduct magnetic flux. It describes how easily a material can be magnetized. For a large value of $\mu_{r}$, a small current $i$ produces a large flux density $B$. The magnetic flux takes the path of the highest permeability.

For ferromagnetic materials, the relationship between $B$ and $H$ is nonlinear because the relative permeability $\mu_{r}$ depends on the magnetic field intensity $H$. Figure 1.1 shows simplified plots of the magnetic flux density $B$ as a function of the magnetic field intensity $H$ for air core inductors and for ferromagnetic core inductors. The straight line describes the air core inductor and has a slope $\mu_{0}$ for all values of $H$. These inductors are linear. The piecewise linear approximation corresponds to the ferromagnetic core inductors, where $B_{s}$ is the saturation flux density and $H_{s}=B_{s} /\left(\mu_{r} \mu_{0}\right)$ is the magnetic field intensity corresponding to $B_{s}$. At low values of the magnetic flux density $B<$ $B_{s}$, the relative permeability $\mu_{r}$ is high and the slope of the $B-H$ curve $\mu_{r} \mu_{0}$ is also high. For $B>B_{s}$, the core saturates and $\mu_{r}=1$, reducing the slope of the $B-H$ curve to $\mu_{0}$.

The total peak magnetic flux density $B_{p k}$, which in general consists of both the dc component $B_{D C}$ and the amplitude of the ac component $B_{m}$, should be lower than the saturation flux density $B_{s}$ of a magnetic core at the highest operating temperature $T_{\max }$ :

$$
B_{p k}=B_{D C}+B_{m} \leq B_{s} .
$$

The dc component of the magnetic flux density is caused by the dc component of the inductor current $I_{L}$ :

$$
B_{D C}=\frac{\mu_{r} \mu_{0} N I_{L}}{l_{c}}
$$

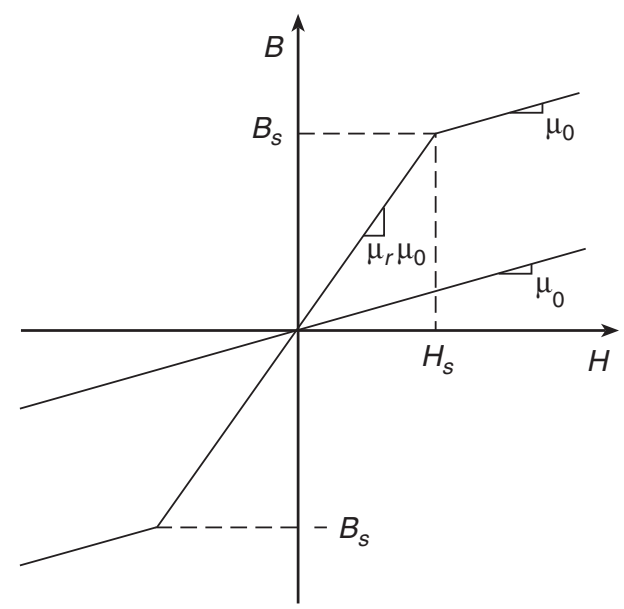

Figure 1.1 Simplified plots of magnetic flux density $B$ as a function of magnetic field intensity $H$ for air core inductors (straight line) and ferromagnetic core inductors (piecewise linear approximation). 
The ac component of the magnetic flux density is caused by the ac component of the inductor current with its amplitude $I_{m}$ :

$$
B_{m}=\frac{\mu_{r} \mu_{0} N I_{m}}{l_{c}}
$$

Hence,

$$
B_{p k}=\frac{\mu_{r} \mu_{0} N I_{L}}{l_{c}}+\frac{\mu_{r} \mu_{0} N I_{m}}{l_{c}}=\frac{\mu_{r} \mu_{0} N\left(I_{L}+I_{m}\right)}{l_{c}} \leq B_{s} .
$$

The saturation flux density $B_{s}$ decreases with temperature. For ferrites, $B_{s}$ may decrease by a factor of 2 as the temperature increases from $20^{\circ} \mathrm{C}$ to $90^{\circ} \mathrm{C}$.

\subsubsection{Magnetic Flux Linkage}

The magnetic flux linkage is the sum of the flux enclosed by each turn of wire wound around the core. The magnetic flux linkage is the magnetic flux linking $N$ turns and is described by

$$
\lambda=N \phi=N A_{c} B=N A_{c} \mu H=\frac{\mu A_{c} N^{2} i}{l_{c}}=L i(\mathrm{~V} \mathrm{~s}) .
$$

This equation is analogous to Ohm's law $v=R i$ and the equation for the capacitor charge $Q=C v$. The unit of the flux linkage is the weber-turn. For sinusoidal waveforms, the relationship among the amplitudes is

$$
\lambda_{m}=N \phi_{m}=N A_{c} B_{m}=N A_{c} \mu H_{m}=\frac{\mu_{r} \mu_{0} A_{c} N^{2} I_{m}}{l_{c}} .
$$

The change in the magnetic linkage can be expressed as

$$
\Delta \lambda=\int_{t_{1}}^{t_{2}} v d t=\lambda\left(t_{2}\right)-\lambda\left(t_{1}\right) .
$$

\subsection{Magnetic Circuits}

\subsubsection{Reluctance}

The reluctance $\mathcal{R}$ is the resistance of the core to the flow of the magnetic flux $\phi$. It opposes the magnetic flux flow, similarly to the way the resistance opposes the electric current flow. An element of a magnetic circuit can be called a reluctor. The concept of reluctance is illustrated in Figure 1.2. The reluctance of a basic magnetic circuit element is given by

$$
\mathcal{R}=\frac{1}{\mathcal{P}}=\frac{l_{c}}{\mu A_{c}}\left(\frac{\mathrm{A} \cdot \text { turns }}{\mathrm{Wb}}\right)=\frac{l_{c}}{\mu A_{c}}\left(\frac{\text { turns }}{\mathrm{H}}\right),
$$

where $A_{c}$ is the cross-sectional area of the core (i.e., the area through which the magnetic flux flows) and $l_{c}$ is the mean magnetic path length (MPL), which is the mean length of the closed path that the magnetic flux flows around the magnetic circuit. The reluctance is directly proportional the length of the magnetic path $l_{c}$ and is inversely proportional to the cross-sectional area $A_{c}$ through which the magnetic flux $\phi$ flows. The permeance of a basic magnetic circuit element is

$$
\mathcal{P}=\frac{1}{\mathcal{R}}=\frac{\mu A_{c}}{l_{c}}\left(\frac{\mathrm{Wb}}{\mathrm{A} \cdot \text { turns }}\right)=\frac{\mu A_{c}}{l_{c}}\left(\frac{\mathrm{H}}{\text { turns }}\right) .
$$

When the number of turns $N=1, L=\mathcal{P}$. The reluctance is the magnetic resistance because it opposes the establishment and the flow of a magnetic flux $\phi$ in a material. A poor conductor of the magnetic flux has a high reluctance and a low permeance. The magnetic Ohm's law is expressed as

$$
\phi=\frac{\mathcal{F}}{\mathcal{R}}=\mathcal{P} \mathcal{F}=\frac{\mu A_{c} N i}{l_{c}}=\frac{\mu_{r c} \mu_{0} A_{c} N i}{l_{c}}(\mathrm{~Wb}) .
$$

Magnetic flux always takes the path with the highest permeability $\mu$. 


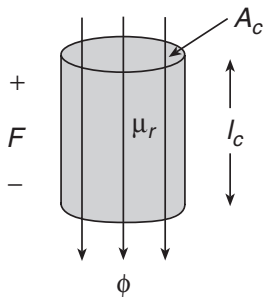

(a)

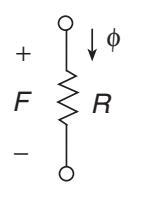

(b)

Figure 1.2 Reluctance. (a) Basic magnetic circuit element conducting magnetic flux $\phi$. (b) Equivalent magnetic circuit.

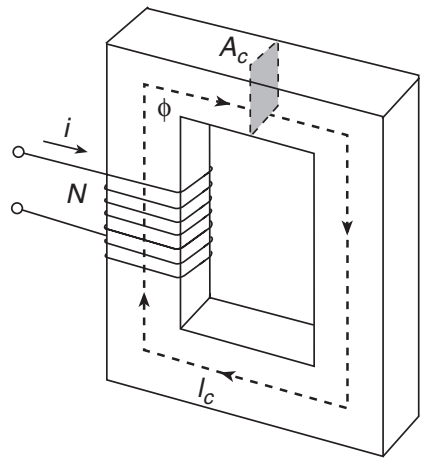

(a)

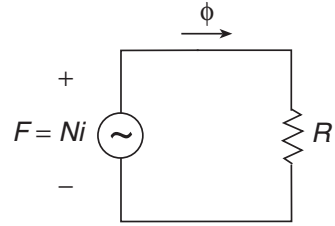

(b)

Figure 1.3 Magnetic circuit. (a) An inductor composed of a core and a winding. (b) Equivalent magnetic circuit.

In general, the magnetic circuit is the space in which the magnetic flux flows around the coil(s). Figure 1.3 shows an example of a magnetic circuit. The reluctance in magnetic circuits is analogous to the resistance $R$ in electric circuits. Likewise, the permeance in magnetic circuits is analogous to the conductance in electric circuits. Therefore, magnetic circuits described by the equation $\phi=\mathcal{F} / \mathcal{R}$ can be solved in a similar manner as electric circuits described by Ohm's law $I=V / R=G V=(\sigma A / l) V$, where $\phi, \mathcal{F}, \mathcal{R}, \mathcal{P}, B, \lambda$, and $\sigma$ correspond to $I, V, R, G, J, Q$, and $\mu$, respectively. For example, the reluctances can be connected in series or in parallel. In addition, the reluctance $\mathcal{R}=l_{c} / \mu A_{c}$ is analogous to electric resistance $R=l / \sigma A$, and flux density $B=\phi / A_{c}$ is analogous to current density $J=I / A$. Table 1.1 lists analogous magnetic and electric quantities.

\subsubsection{Magnetic Kirchhoff's Voltage Law}

Physical structures made of magnetic devices, such as inductors and transformers, can be analyzed just like electric circuits. The magnetic law, analogous to Kirchhoff's voltage law, states that the sum of the magnetomotive forces $\mathcal{F}_{k}$ and the magnetic potential differences $\mathcal{R}_{k} \phi_{k}$ around the closed magnetic loop is zero:

$$
\sum_{k=1}^{n} \mathcal{F}_{k}-\sum_{k=1}^{m} \mathcal{R}_{k} \phi_{k}=0 .
$$

For instance, an inductor with a simple core having an air gap as illustrated in Figure 1.4 is given by

$$
N i=\mathcal{F}=\mathcal{F}_{c}+\mathcal{F}_{g}=\phi\left(\mathcal{R}_{c}+\mathcal{R}_{g}\right)
$$


Table 1.1 Analogy between magnetic and electric quantities

\begin{tabular}{ll}
\hline Magnetic quantity & Electric quantity \\
\hline $\mathcal{F}=N i$ & $V$ \\
$\phi$ & $I$ \\
$H$ & $E$ \\
$B$ & $J$ \\
$\mathcal{P}$ & $G$ \\
$\lambda$ & $Q$ \\
$\mu$ & $\sigma$ \\
$L$ & $C$ \\
$\phi=\frac{\mathcal{F}}{\mathcal{R}}$ & $I=\frac{V}{R}$ \\
$B=\frac{\phi}{A}$ & $J=\frac{I}{A}$ \\
$H=\frac{\mathcal{F}}{l}=\frac{N i}{l}$ & $E=\frac{V}{l}$ \\
$\mathcal{R}=\frac{l}{\mu A}$ & $R=\frac{l}{\sigma A}$ \\
$B=\mu H$ & $D=\epsilon E$ \\
$\lambda=L i$ & $Q=C v$ \\
$w_{m}=\frac{1}{2} \mu H^{2}$ & $w_{e}=\frac{1}{2} \epsilon E^{2}$ \\
$W_{m}=\frac{1}{2} L i^{2}$ & $W_{e}=\frac{1}{2} C v^{2}$ \\
\hline
\end{tabular}

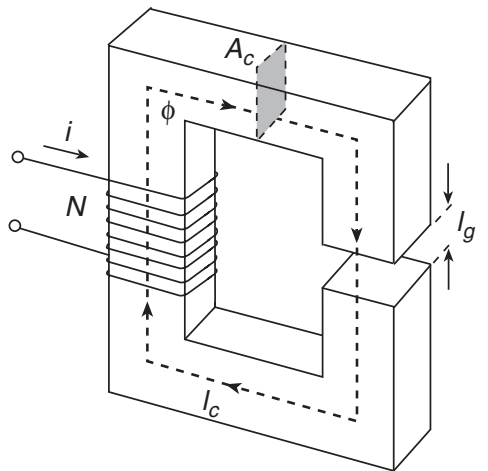

(a)

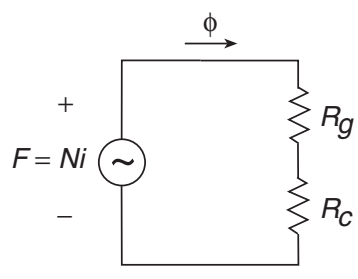

(b)

Figure 1.4 Magnetic circuit illustrating the magnetic Kirchhoff's voltage law. (a) An inductor composed of a core with air gap and a winding. (b) Equivalent magnetic circuit.

where the reluctance of the core is

$$
\mathcal{R}_{c}=\frac{l_{c}}{\mu_{r c} \mu_{0} A_{c}}
$$

the reluctance of the air gap is

$$
\mathcal{R}_{g}=\frac{l_{g}}{\mu_{0} A_{c}},
$$

and it assumed that $\phi_{c}=\phi_{g}=\phi$. The reluctance of the air gap $\mathcal{R}_{g}$ is much higher than the reluctance of the core $\mathcal{R}_{c}$. 


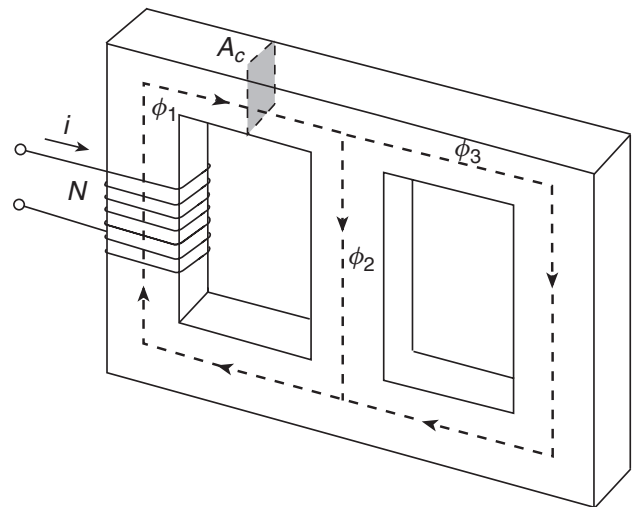

(a)

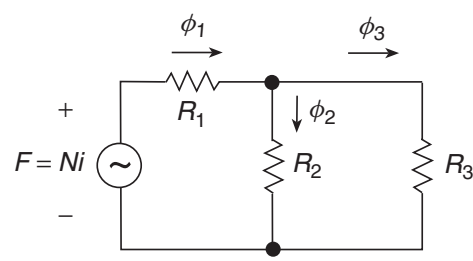

(b)

Figure 1.5 Magnetic circuit illustrating the continuity of the magnetic flux. (a) An inductor composed of a core and a winding. (b) Equivalent magnetic circuit.

\subsubsection{Magnetic Flux Continuity}

The continuity of the magnetic flux law states that the net magnetic flux through any closed surface is always zero,

$$
\phi=\oiint_{A} B d A=0
$$

or the net magnetic flux entering the node is zero,

$$
\sum_{k=1}^{n} \phi_{k}=\sum_{k=1}^{n} A_{k} B_{k}=0 .
$$

This law is analogous to Kirchhoff's current law introduced by Gauss and can be called Kirchhoff's flux law. Figure 1.5 illustrates the continuity of the magnetic flux law. For example, when three core legs meet at a node,

$$
\phi_{1}=\phi_{2}+\phi_{3},
$$

which can be expressed by

$$
\frac{\mathcal{F}_{1}}{\mathcal{R}_{1}}=\frac{\mathcal{F}_{2}}{\mathcal{R}_{2}}+\frac{\mathcal{F}_{3}}{\mathcal{R}_{3}} .
$$

If all three legs of the core have windings, then we have

$$
\frac{N_{1} i_{1}}{\mathcal{R}_{1}}=\frac{N_{2} i_{2}}{\mathcal{R}_{2}}+\frac{N_{3} i_{3}}{\mathcal{R}_{3}}
$$

Usually, most of the magnetic flux is confined inside an inductor, e.g., for an inductor with a toroidal core. The flux outside an inductor is called the leakage flux.

\subsection{Magnetic Laws}

\subsubsection{Ampère's Law}

Ampère's law (1826) states that a time-varying current $i(t)$ induces a time-varying magnetic field $H(t)$. When a conductor (such as an inductor) carries a time-varying current $i(t)$, a magnetic field $H(t)$ is induced. In a conductor, the induced magnetic field may be due to the conductor's own ac 
current or the ac current of other adjacent conductors. The integral form of Ampère's circuital law, or simply Ampère's law, states that the closed line integral of the magnetic field intensity $\mathbf{H}$ around a closed path $C$ is equal to the total current $i_{\text {enc }}$ passing through the interior of the closed path bounding the surface $S$ :

$$
\oint_{C} \mathbf{H} \cdot d \mathbf{l}=\iint_{S} \mathbf{J} \cdot d \mathbf{S}=\sum_{n=1}^{N} i_{n}=i_{1}+i_{2}+\cdots+i_{N}=i_{e n c},
$$

where $d \mathbf{l}$ is the vector length element pointing in the direction of the Amperian path. The current $i_{\text {enc }}$ enclosed by the path $C$ is given by the integral of the normal component $\mathbf{J}$ over the surface $S$. The surface integral of the current density $\mathbf{J}$ is equal to the conduction current $I$ flowing through the surface $S$. For good conductors, the displacement current can be ignored. For an inductor with $N$ turns, Ampère's law is

$$
\oint_{C} \mathbf{H} \cdot d \mathbf{l}=N i
$$

Ampère's law in its discrete form can be expressed as

$$
\sum_{k=1}^{n} H_{k} l_{k}=\sum_{k=1}^{m} N_{k} i_{k}
$$

For example, Ampère's law for an inductor with an air gap is

$$
H_{c} l_{c}+H_{g} l_{g}=N i
$$

If the current density $J$ is uniform and perpendicular to the surface $S$,

$$
H C=S J .
$$

The current density $J$ in winding conductors of magnetic components used in power electronics is usually in the range of 0.1 to $10 \mathrm{~A} / \mathrm{mm}^{2}$. The displacement current is neglected in (1.27). Ampère's law constitutes one of Maxwell's equations in integral form.

\section{Example 1.1}

An infinitely long round straight wire of radius $r_{o}$ carries current $i=I_{m} \cos \omega t$ in steady state at low frequencies. Determine the waveforms of the magnetic field intensity $H(r, t)$ inside and outside the wire.

Solution: At low frequencies, the skin effect can be neglected and the current is uniformly distributed over the cross section of the wire, as shown in Figure 1.6.

The magnetic field intensity inside the wire. The current in the conductor induces a concentric magnetic field inside and outside the conductor. The current flowing through the area enclosed by the cylindrical shell of radius $r$ at low frequencies is given by

$$
i_{\text {enc }}=I_{m(\text { enc })} \cos \omega t
$$

where $I_{m(e n c)}$ is the amplitude of the current enclosed by the shell. Hence, the amplitude of the current density at a radius $r$ is

$$
J_{m}(r)=\frac{I_{m(e n c)}}{\pi r^{2}}, \quad \text { for } r \leq r_{o},
$$

and the amplitude of the current density at the wire surface $r=r_{o}$ is

$$
J_{m}\left(r_{o}\right)=\frac{I_{m}}{\pi r_{o}^{2}} .
$$




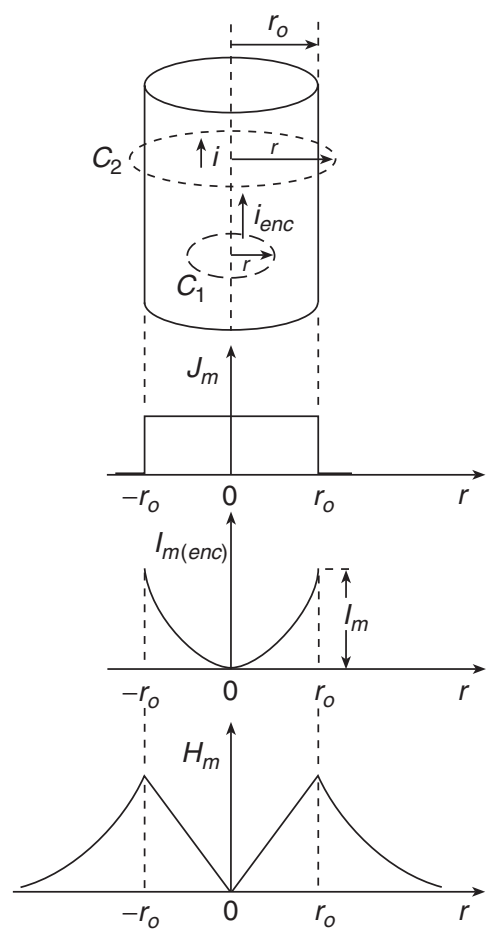

Figure 1.6 Cross section of an infinitely long round straight wire carrying a uniform current $i$ and amplitudes of current density $J_{m}$, enclosed current $I_{m(e n c)}$, and magnetic field intensity $H_{m}$ as a function of the distance from the wire center $r$ at low frequencies (i.e., when the skin effect can be neglected).

The current density is uniform at low frequencies (where the skin effect can be neglected), i.e., $J_{m}(r)=J_{m}\left(r_{o}\right)$, yielding the amplitude of the enclosed current

$$
I_{m(\text { enc })}=I_{m}\left(\frac{r}{r_{o}}\right)^{2}, \quad \text { for } r \leq r_{o} .
$$

From Ampère's law,

$$
I_{m(e n c)}=\oint_{C_{1}} \mathbf{H} \cdot d \mathbf{l}=H_{m}(r) \oint_{C_{1}} d l=2 \pi r H_{m}(r), \quad \text { for } r \leq r_{o},
$$

where $C_{1}=2 \pi r$ with $r \leq r_{o}$. Figure 1.6 shows a plot of $I_{m(e n c)}$ as a function of distance from the conductor center $r$. Equating the right-hand sides of (1.35) and (1.36), we obtain the amplitude of the magnetic field intensity inside the wire for low frequencies

$$
H_{m}(r)=I_{m}\left(\frac{r}{r_{o}}\right)^{2} \frac{1}{2 \pi r}=I_{m} \frac{r}{2 \pi r_{o}^{2}}, \text { for } r \leq r_{o} .
$$

Figure 1.6 shows a plot of the amplitude of the magnetic field intensity $H_{m}$ as a function of $r$. The amplitude of the magnetic field intensity $H_{m}$ is zero at the wire center because the enclosed current is zero. The waveform of the magnetic field inside the wire at low frequencies is given by

$$
H(r, t)=I_{m} \frac{r}{2 \pi r_{o}^{2}} \cos \omega t, \quad \text { for } r \leq r_{o} .
$$

Thus, the amplitude of the magnetic field intensity $H_{m}$ inside the wire at radius $r$ is determined solely by the amplitude of the current inside the radius $r$. 
The magnetic field intensity outside the wire. The entire current $i=I_{m} \cos \omega t$ is enclosed by a path of radius $r \geq r_{o}$. From Ampère's law, the amplitude of the entire current $i$ is

$$
I_{m}=\oint_{C_{2}} \mathbf{H} \cdot d \mathbf{l}=H_{m}(r) \oint_{C_{2}} d l=2 \pi r H_{m}(r), \quad \text { for } r \geq r_{o},
$$

where $C_{2}=2 \pi r$ with $r \geq r_{o}$. The amplitude of the magnetic field intensity outside the conductor at any frequency is given by the expression

$$
H_{m}(r)=\frac{I_{m}}{2 \pi r}, \quad \text { for } r \geq r_{o},
$$

and the waveform of this field is

$$
H(r, t)=\frac{I_{m}}{2 \pi r} \cos \omega t, \quad \text { for } r \geq r_{o} .
$$

The amplitude of the magnetic field intensity increases linearly with $r$ inside the wire from 0 to $H_{m}\left(r_{o}\right)=I_{m} /\left(2 \pi r_{o}\right)$ at low frequencies. The amplitude of the magnetic field intensity is inversely proportional to $r$ outside the wire at any frequency.

\subsubsection{Faraday's Law}

A time-varying current produces a magnetic field, and a time-varying magnetic field can produce an electric current. In 1820, Hans Christian Oersted showed that a conductor carrying a current produces a magnetic field, which can affect a compass needle. Ampère measured that this magnetic field intensity is linearly related to the current which produces it. In 1831, Michael Faraday discovered that a current can be produced by an alternating magnetic field and that a time-varying magnetic field can induce a voltage, or an electromotive force, in an adjacent circuit. This voltage is proportional to the rate of change of magnetic flux linkage $\lambda$, or magnetic flux $\phi$, or the current $i$, producing the magnetic field.

Faraday's law (1831) states that a time-varying magnetic flux $\phi(t)$ passing through a closed loop, such as an inductor turn, generates a voltage $v(t)$ in the loop and for a linear inductor it is expressed by

$$
\begin{aligned}
v(t)=\frac{d \lambda}{d t} & =\frac{d(N \phi)}{d t}=N \frac{d \phi}{d t}=N \frac{d}{d t}\left(\frac{N i}{\mathcal{R}}\right)=\frac{N^{2}}{\mathcal{R}} \frac{d i}{d t}=L \frac{d i}{d t} \\
& =N A \frac{d B}{d t}=N A \mu \frac{d H}{d t}=\frac{\mu A N^{2}}{l} \frac{d i}{d t} .
\end{aligned}
$$

The voltage $v$ is proportional to the rate of change of current $i$. This voltage, in turn, may produce a current in the circuit. The inductance $L$ relates the induced voltage $v$ to the current $i$. The voltage $v$ across the terminals of an inductor $L$ is proportional to the time rate of change of the current $i$ in the inductor. If the inductor current is constant, the voltage across an ideal inductor is zero. The inductor behaves as a short circuit for a dc current. The inductor current cannot change instantaneously.

For sinusoidal waveforms, the derivative $d / d t$ can be replaced by $j \omega$ and Faraday's law in phasor form can be expressed as

$$
\mathbf{V}_{\mathbf{L m}}=j \omega \lambda_{\mathbf{m}} .
$$

For nonlinear, time-varying inductors, the relationships are

$$
\lambda(t)=L(t) i(t)
$$

and

$$
v(t)=L(t) \frac{d i(t)}{d t}+i(t) \frac{d L(t)}{d t} .
$$

The impedance of lossless inductive components in terms of phasors of sinusoidal inductor current $\mathbf{I}_{\mathbf{L m}}$ and voltage $\mathbf{V}_{\mathbf{m}}=j \omega \boldsymbol{\lambda}_{\mathbf{m}}$ is

$$
Z=\frac{\mathbf{V}_{\mathbf{m}}}{\mathbf{I}_{\mathbf{L m}}}=\frac{j \omega \lambda}{I_{m}}=j \omega L
$$


The impedance of lossy inductive components in terms of phasors is

$$
Z=\frac{\mathbf{V}_{\mathbf{m}}}{\mathbf{I}_{\mathbf{m}}}=\frac{j \omega \lambda_{m}}{I_{m}}=R+j \omega L
$$

Since

$$
v d t=L\left(\frac{d i}{d t}\right) d t=L d i
$$

the current in an inductor is given by

$$
i(t)=\frac{1}{L} \int_{0}^{t} v d t+i(0)=\frac{1}{\omega L} \int_{0}^{\omega t} v d(\omega t)+i(0) .
$$

\subsubsection{Lenz's Law}

Lenz's law (1834) states that the voltage $v(t)$ induced by an applied time-varying magnetic flux $\phi_{a}(t)$ has a direction that induces current $i_{E}(t)$ in the closed loop, which in turn induces a magnetic flux $\phi_{i}(t)$ that tends to oppose the change in the applied flux $\phi_{a}(t)$, as illustrated in Figure 1.7. The direction of the induced current is always such that it produces a magnetic field that opposes the change in the original flux. If $\phi_{a}(t)$ increases, the induced current produces an opposing flux $\phi_{i}(t)$. If $\phi_{a}(t)$ decreases, the induced current produces an aiding flux $\phi_{i}(t)$. The induced currents in the closed loops are called eddy currents. Eddy currents occur when a conductor is subjected to time-varying magnetic fields. In accordance with Lenz's law, the eddy currents produce their own magnetic fields to oppose the original field. The effects of eddy currents on winding conductors and magnetic cores are: nonuniform current distribution, increased effective resistance, increased power loss, and reduced internal inductance. If the resistivity of a conductor was zero (as in a perfect conductor), eddy-current loops would be generated with such a magnitude and phase to exactly cancel the applied magnetic field. A perfect conductor would oppose any change in externally applied magnetic field. Circulating eddy currents would be induced to oppose any buildup of magnetic field in the conductor.

\subsubsection{Ohms's Law}

The point form of Ohm's law (1827) for conducting materials is

$$
\mathbf{E}=\rho \mathbf{J}=\frac{\mathbf{J}}{\sigma},
$$

where $\rho$ is the resistivity and $\sigma=1 / \rho$ is the conductivity of a material.

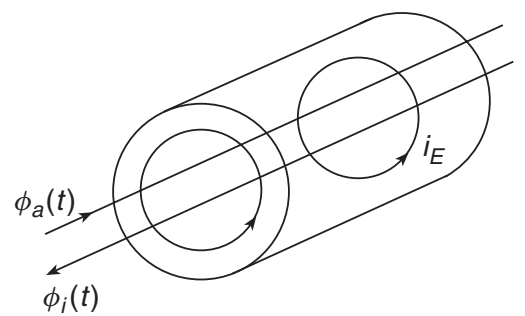

Figure 1.7 Illustration of Lenz's law generating eddy currents. The applied time-varying magnetic flux $\phi_{a}(t)$ induces eddy current $i_{E}(t)$, which in turn generates induced flux $\phi_{i}(t)$ that opposes changes in the applied flux $\phi_{a}(t)$. 


\subsubsection{Maxwell's Equations}

Maxwell's equations (1865) govern electromagnetic waves. They couple electric fields, magnetic fields, and current densities. Maxwell's equations in differential (point) form in the time domain at any point in space and at any time are given by

$$
\begin{aligned}
\nabla \times \mathcal{H} & =\mathcal{J}+\frac{\partial \mathcal{D}}{\partial t} \quad \text { (Ampère's law) } \\
\nabla \times \mathcal{E} & =-\frac{\partial \mathcal{B}}{\partial t}=-\mu \frac{\partial \mathcal{H}}{\partial t} \quad \text { (Faraday's law) } \\
\nabla \cdot \mathcal{D} & =\rho_{v} \quad(\text { Gauss's law), } \\
\nabla \cdot \mathcal{B} & =0 \quad \text { (flux continuity law). }
\end{aligned}
$$

where $\partial \mathcal{D} / \partial t$ is the displacement current density. The current density $\mathcal{J}$ and the volume charge density $\rho_{v}$ are the sources of electromagnetic fields $\mathcal{H}, \mathcal{B}=\mu \mathcal{H}, \mathcal{E}$, and $\mathcal{D}=\epsilon \mathcal{E}$, where $\mu$ is the permeability and $\epsilon$ is the permittivity. They are related by the charge or current conservation equation

$$
\nabla \cdot \mathcal{J}+\frac{\partial \rho_{v}}{\partial t}=0
$$

Script letters are used to designate instantaneous field quantities, which are functions of position and time, e.g., $\mathcal{E}(x, y, z, t)$.

The phasor technique is a useful mathematical tool for solving problems in linear systems that involve periodic sinusoidal or nonsinusoidal waveforms in steady state. A periodic nonsinusoidal waveform, such as the rectangular wave, can be expanded into a Fourier series of sinusoidal components, which is a superposition of harmonic sinusoids. If the excitation is a sinusoidal function of time, the steady-state waveforms described in the time domain can be represented by phasors and integro-differential equations become linear equations with no sinusoidal functions, which are easy to solve. Differentiation in the time domain is equivalent to multiplication by $j \omega$ in the phasor domain, and integration in the time domain is equivalent to division by $j \omega$ in the phasor domain. The solutions in the phasor domain can be converted back into the time domain.

The electric field intensity for the one-dimensional case in the time domain is given by

$$
\mathcal{E}(x, t)=E_{m}(0) e^{-\frac{x}{\delta_{w}}} \cos \left(\omega t-\frac{x}{\delta_{w}}+\phi_{o}\right)=\operatorname{Re}\left\{\mathbf{E}(x) e^{j \omega t}\right\},
$$

where $\delta_{w}$ is the skin depth and the phasor of the electric field intensity is

$$
\mathbf{E}(x)=E_{m}(0) e^{-\frac{x}{\delta_{w}}} e^{-j \frac{x}{\delta_{w}}} e^{j \phi_{o}} .
$$

Similarly, the magnetic field intensity is

$$
\mathcal{H}(x, t)=H_{m}(0) e^{-\frac{x}{\delta_{w}}} \cos \left(\omega t-\frac{x}{\delta_{w}}+\theta_{o}\right)=\operatorname{Re}\left\{\mathbf{H}(x) e^{j \omega t}\right\},
$$

where the phasor of the magnetic field intensity is

$$
\mathbf{H}(x)=H_{m}(0) e^{-\frac{x}{\delta_{w}}} e^{-j \frac{x}{\delta_{w}}} e^{j \theta_{o}} .
$$

Substituting the electric and magnetic field intensities into Maxwell's equation in the time domain, we obtain

$$
\nabla \times \operatorname{Re}\left\{\mathbf{E}(x) e^{j \omega t}\right\}=-\frac{\partial}{\partial t} \operatorname{Re}\left\{\mu \mathbf{H}(x) e^{j \omega t}\right\}
$$

which becomes

$$
\operatorname{Re}\left\{\nabla \times \mathbf{E}(x) e^{j \omega t}\right\}=\operatorname{Re}\left\{-j \omega \mu \mathbf{H}(x) e^{j \omega t}\right\}
$$


Thus, $\frac{\partial}{\partial t}$ in Maxwell's equations in the time domain can be replaced by $j \omega$ to obtain Maxwell's equations for sinusoidal field waveforms in phasor form:

$$
\begin{aligned}
\nabla \times \mathbf{E} & =-j \omega \mu \mathbf{H}=-j \omega \mathbf{B}, \\
\nabla \times \mathbf{H} & =\mathbf{J}+j \omega \mathbf{D}=(\sigma+j \omega \epsilon) \mathbf{E}, \\
\nabla \cdot \mathbf{D} & =\rho_{v}, \\
\nabla \cdot \mathbf{B} & =0 .
\end{aligned}
$$

The constitutive equations for linear and isotropic materials are:

$$
\begin{aligned}
\mathbf{B} & =\mu \mathbf{H}, \\
\mathbf{D} & =\epsilon \mathbf{H}, \\
\mathbf{J} & =\sigma \mathbf{E} .
\end{aligned}
$$

\subsubsection{Maxwell's Equations for Good Conductors}

In general, Maxwell's equation in phasor form, which is the differential form of Ampère's equation, together with Ohm's law $(\mathbf{J}=\sigma \mathbf{E})$, is given by

$$
\nabla \times \mathbf{H}=\mathbf{J}+j \omega \mathbf{D}=\sigma \mathbf{E}+j \omega \epsilon \mathbf{E}=(\sigma+j \omega \epsilon) \mathbf{E},
$$

where $\sigma$ is the conductivity. For good conductors, conduction current density $\mathbf{J}=\sigma \mathbf{E}$ dominates displacement current density $j \omega \in \mathbf{E}$, and the following inequality is satisfied:

$$
\sigma \gg \omega \epsilon .
$$

For copper, this inequality is satisfied for frequencies up to $10^{16} \mathrm{~Hz}$. Maxwell's equation for good conductors becomes

$$
\nabla \times \mathbf{H} \approx \mathbf{J} .
$$

Maxwell's equation in phasor form, which is the differential form of Faraday's law, is expressed as

$$
\nabla \times \mathbf{E}=-j \omega \mu \mathbf{H} .
$$

Using Ohm's law $\mathbf{E}=\mathbf{J} / \sigma$, we obtain

$$
\nabla \times \frac{\mathbf{J}}{\sigma}=-j \omega \mu \mathbf{H}
$$

producing another form of Maxwell's equation,

$$
\nabla \times \mathbf{J}=-j \omega \mu \sigma \mathbf{H} .
$$

Assuming that $\sigma$ and $\mu$ are homogeneous, taking the curl on both sides of the above equation, and substituting into Maxwell's equation,

$$
\nabla \times(\nabla \times \mathbf{J})=-j \omega \mu \sigma \nabla \times \mathbf{H}=-j \omega \mu \sigma \mathbf{J} .
$$

Expanding the left-hand side,

$$
\nabla(\nabla \cdot \mathbf{J})-\nabla^{2} \mathbf{J}=-j \omega \mu \sigma \mathbf{J},
$$

where the principle of conservation of charge states that charge can be neither created nor destroyed, and its point form is expressed by $\boldsymbol{\nabla} \cdot \mathbf{J}=0$. It is a point form of Kirchhoff's current law. The conduction current density $\mathbf{J}$ in good conductors must satisfy the second-order partial differential equation

$$
\nabla^{2} \mathbf{J}=j \omega \mu \sigma \mathbf{J} .
$$




\section{4 HIGH-FREQUENCY MAGNETIC COMPONENTS}

For good conductors,

$$
\nabla \cdot(\nabla \times \mathbf{H})=(\sigma+j \omega \epsilon)(\nabla \cdot \mathbf{E})=0 .
$$

Hence, Maxwell's equation for good conductors becomes

$$
\nabla \cdot \mathbf{D}=\rho_{v}=0 .
$$

In good conductors, mobile electrons drift through a lattice of positive ions encountering frequent collisions. On average, the net charge over a large volume (compared with atomic dimensions) is zero even though some of the charges are moving and causing current flow. The net movement velocity or drift velocity is proportional to the electric field intensity.

\subsubsection{Poynting Vector}

The instantaneous Poynting vector (1884) at a given point is expressed by

$$
\mathcal{S}=\mathcal{E} \times \mathcal{H} \quad\left(\mathrm{W} / \mathrm{m}^{2}\right) .
$$

The Poynting vector represents the direction and density of power flow at any point, i.e., it is the rate at which energy flows through a unit surface area perpendicular to direction of wave propagation. The direction of $\mathcal{S}$ is normal to both $\mathcal{E}$ and $\mathcal{H}$. The cross product $\mathbf{E} \times \mathbf{H}$ points in the direction of power flow. The vector $\mathcal{S}$ represents an instantaneous surface power density, i.e., an instantaneous power per unit area. Since $\mathcal{E}$ is measured in $\mathrm{V} / \mathrm{m}$ and $\mathcal{H}$ in $\mathrm{A} / \mathrm{m}, \mathcal{S}$ is measured in $(\mathrm{V} / \mathrm{m}) \times(\mathrm{A} / \mathrm{m})$ $=\mathrm{VA} / \mathrm{m}^{2}=\mathrm{W} / \mathrm{m}^{2}$.

For time-harmonic fields, the complex Poynting vector is

$$
\mathbf{S}_{c}=\mathbf{E} \times \mathbf{H}^{*} \quad\left(\mathrm{~W} / \mathrm{m}^{2}\right) .
$$

The time-averaged power density (i.e., averaged over one period of the sinusoidal excitation) is given by the time-averaged Poynting vector

$$
\mathbf{S}_{a v}=\frac{1}{2} \operatorname{Re}\left\{\mathbf{E} \times \mathbf{H}^{*}\right\} \quad\left(\mathrm{W} / \mathrm{m}^{2}\right) .
$$

The amount of time-averaged power passing through a surface $S$ is

$$
P_{a v}=\int_{S} \mathbf{S}_{a v} \cdot d \mathbf{s}=\frac{1}{2} \operatorname{Re}\left\{\int_{s}\left(\mathrm{E} \times \mathrm{H}^{*}\right) \bullet d \mathrm{~s}\right\} \quad(\mathrm{W}) .
$$

For a linear, isotropic, and time-invariant medium, the Poynting theorem is

$$
\oint_{S}(\mathbf{E} \times \mathbf{H}) \cdot d \mathbf{s}=-\int_{V} \mathbf{J} \cdot \mathbf{E} d V-\frac{\partial}{\partial t} \int_{V}\left(\frac{1}{2} \mu H^{2}+\frac{1}{2} \epsilon E^{2}\right) d V .
$$

For sinusoidal field waveforms,

$$
\begin{aligned}
\oint_{S}\left(\mathbf{E} \times \mathbf{H}^{*}\right) \cdot d \mathbf{s} & =-\int_{V}\left[\mathbf{E} \cdot \mathbf{J}^{*}+j \omega\left(\mathbf{H}^{*} \cdot \mathbf{B}+\mathbf{E}^{*} \cdot \mathbf{D}\right] d V\right. \\
& =-\frac{1}{2} \int_{V} \rho J^{2} d V-j \omega \int_{V}\left(\frac{1}{2} \mu H^{2}+\frac{1}{2} \epsilon E^{2}\right) d V \\
& =-\int_{V} p_{d} d V-\frac{\partial}{d \partial} \int_{V}\left(w_{m}+w_{e}\right) d V
\end{aligned}
$$

where an asterisk * indicates a complex conjugate quantity, $\mathbf{E}=\rho \mathbf{J}$ is point Ohm's law, $w_{m}=\frac{1}{2} \mu H^{2}$ is the point magnetic energy density, and $w_{e}=\frac{1}{2} \epsilon E^{2}$ is the point electric energy density. The first term on the right-hand side of (1.85) represents the ohmic power dissipated as heat in the volume $V$ as a result of the flow of conduction current density $\mathbf{J}=\sigma \mathbf{E}$ due to the presence of the electric field $\mathbf{E}$. This power exits the volume $V$ through its surface $S$. The second and third terms represent 
the time rate of change (decrease) of the magnetic and electric energies stored in the magnetic and electric fields, respectively. The principle of conservation of energy states in this case that the total power flow out of a closed surface $S$ at any instant is equal to the sum of the ohmic power dissipated within the enclosed volume $V$ and the rates of decrease of the stored magnetic and electric energies.

In steady state, the complex power flowing into a volume $V$ surrounded by a closed surface $S$ is given by

$$
P=\frac{1}{2} \oint_{S}\left(\mathbf{E} \times \mathbf{H}^{*}\right) \cdot d \mathbf{s}=P_{D}+2 j \omega\left(W_{m}+W_{e}\right) \quad(\mathrm{W}),
$$

where the time-averaged real power dissipated in the volume $V$ is given by Joule's law as

$$
P_{D}=\frac{1}{2} \int_{V} \mathbf{E} \cdot \mathbf{J}^{*} d V=\frac{1}{2} \int_{V} \rho|\mathbf{J}|^{2} d V=\frac{1}{2} \int_{V} \sigma|\mathbf{E}|^{2} d V .
$$

$w_{m}=\mu H^{2} / 2\left(\mathrm{~J} / \mathrm{m}^{3}\right)$ is the magnetic energy density stored in the magnetic field in the volume $V$, $w_{e}=\epsilon E^{2} / 2\left(\mathrm{~J} / \mathrm{m}^{3}\right)$ is the electric energy density stored in the electric field in the volume $V, \mathbf{B} \cdot \mathbf{H} / 2$ is the magnetic energy density, $\mathbf{D} \cdot \mathbf{E} / 2$ is the electric energy density, and $p_{D}=\rho J^{2}\left(\mathrm{~W} / \mathrm{m}^{3}\right)$ is the ohmic power loss density.

\subsubsection{Joule's Law}

The current density in a conductor in the time domain in steady state for the one-dimensional case is described by

$$
\operatorname{Re}\left\{\mathbf{J}(x) e^{j \omega t}\right\}=J(x, t)=J_{m}(0) e^{-\frac{x}{\delta_{w}}} \cos \left(\omega t-\frac{x}{\delta_{w}}+\phi_{o}\right),
$$

where $\delta_{w}$ is the skin depth and $\phi_{o}$ is the initial phase. It is assumed that the current amplitude varies only in the $x$ direction. From Ohm's law,

$$
E(x, t)=\rho J(x, t)=\rho J_{m}(0) e^{-\frac{x}{\delta_{w}}} \cos \left(\omega t-\frac{x}{\delta_{w}}+\phi_{o}\right),
$$

where $E_{m}(0)=\rho J_{m}(0)$. Assuming that $\rho$ is a real number, the phase shift between $J(x, t)$ and $E(x, t)$ is zero. The instantaneous power density at a point is given by

$$
\begin{aligned}
p(x, t) & =J(x, t) E(x, t)=J_{m}(0) E_{m}(0) e^{-\frac{2 x}{\delta_{w}}} \cos ^{2}\left(\omega t-\frac{x}{\delta_{w}}+\phi_{o}\right) \\
& =\rho J_{m}^{2}(0) e^{-\frac{2 x}{\delta_{w}}} \cos ^{2}\left(\omega t-\frac{x}{\delta_{w}}+\phi_{o}\right) \\
& =\frac{J_{m}(0) E_{m}(0)}{2} e^{-\frac{2 x}{\delta_{w}}}+\frac{J_{m}(0) E_{m}(0)}{2} e^{-\frac{2 x}{\delta_{w}}} \cos 2\left(\omega t-\frac{x}{\delta_{w}}+\phi_{o}\right) \\
& =\frac{\rho J_{m}^{2}(0)}{2} e^{-\frac{2 x}{\delta_{w}}}+\frac{\rho J_{m}^{2}(0)}{2} e^{-\frac{2 x}{\delta_{w}}} \cos 2\left(\omega t-\frac{x}{\delta_{w}}+\phi_{o}\right) \\
& =p_{D}(x)+p_{D}(x) \cos 2\left(\omega t-\frac{x}{\delta_{w}}+\phi_{o}\right),
\end{aligned}
$$

where $\cos ^{2} z=\frac{1}{2}+\frac{1}{2} \cos 2 z$. The first term in the above equation represents the time-averaged real power density dissipated in a conductor at a point, and the second term represents the ac component of the instantaneous real power density dissipated in a conductor as heat at a point. The time-averaged real power density dissipated in a conductor at a point is

$$
p_{D}(x)=\frac{1}{T} \int_{0}^{T} p(x, t) d t=\frac{1}{2 \pi} \int_{0}^{2 \pi} p(x, \omega t) d(\omega t)=\frac{J_{m}(0) E_{m}(0)}{2} e^{-\frac{2 x}{\delta_{w}}}=\frac{\rho J_{m}^{2}(0)}{2} e^{-\frac{2 x}{\delta_{w}}},
$$




\section{6}

where $T$ is the period. The total time-averaged power dissipated as heat in a conductor of volume $V$ is

$$
\begin{aligned}
P_{D} & =\iiint_{V} p_{D}(x) d V=\frac{1}{2} \iiint_{V} J_{m}(0) E_{m}(0) e^{-\frac{2 x}{\delta_{w}}} d x d y d z \\
& =\frac{1}{2} \iiint_{V} \rho J_{m}^{2}(0) e^{-\frac{2 x}{\delta_{w}}} d x d y d z .
\end{aligned}
$$

When electromagnetic fields are sinusoidal, phasors are described in space as follows: $\mathbf{H}(\mathbf{r})=$ $\mathbf{H}(x, y, z), \mathbf{E}(\mathbf{r})=\mathbf{E}(x, y, z)$, and $\mathbf{J}(\mathbf{r})=\mathbf{J}(x, y, z)$. The point (local) power density is

$$
\begin{aligned}
p(\mathbf{r}, t) & =\operatorname{Re}\left\{\mathbf { J } ( \mathbf { r } , t \} \cdot \operatorname { R e } \left\{\mathbf{E}(\mathbf{r}, t\}=\frac{1}{4}\left[\mathbf{J}(\mathbf{r}, t)+\mathbf{J}^{*}(\mathbf{r}, t)\right]\left[\mathbf{E}(\mathbf{r}, t)+\mathbf{E}^{*}(\mathbf{r}, t)\right]\right.\right. \\
& =\frac{1}{4}\left[\mathbf{J}(\mathbf{r}) \cdot \mathbf{E}^{*}(\mathbf{r})+\mathbf{J}(\mathbf{r}) \cdot \mathbf{E}(\mathbf{r}) e^{2 j \omega t}+\mathbf{J}^{*}(\mathbf{r}) \cdot \mathbf{E}(\mathbf{r})+\mathbf{J}^{*}(\mathbf{r}) \cdot \mathbf{E}^{*}(\mathbf{r}) e^{-2 j \omega t}\right] \\
& =\frac{1}{2} \operatorname{Re}\left[\mathbf{J}(\mathbf{r}) \cdot \mathbf{E}^{*}(\mathbf{r})+\mathbf{J}(\mathbf{r}) \cdot \mathbf{E}(\mathbf{r}) e^{2 j \omega t}\right] .
\end{aligned}
$$

The time-averaged real power density dissipated in a conductor at a point $\mathbf{r}$ is

$$
p_{D}(\mathbf{r})=\frac{1}{T} \int_{0}^{T} p(\mathbf{r}, t) d t=\frac{1}{2} \operatorname{Re}\left[\mathbf{J}(\mathbf{r}) \cdot \mathbf{E}^{*}(\mathbf{r})\right] .
$$

The time-averaged power dissipated as heat in the conductor of volume $V$ is given by

$$
\begin{aligned}
P_{D} & =\iiint_{V} p_{D}(\mathbf{r}) d V=\frac{1}{2} \operatorname{Re} \iiint_{V} \mathbf{J}(\mathbf{r}) \cdot \mathbf{E}^{*}(\mathbf{r}) d V \\
& =\frac{1}{2} \iiint_{V} \rho \mathbf{J}(\mathbf{r}) \cdot \mathbf{J}^{*}(\mathbf{r}) d V=\frac{1}{2} \iiint_{V} \rho|\mathbf{J}(\mathbf{r})|^{2} d V .
\end{aligned}
$$

The current density in phasor form for the one-dimensional case is given by

$$
\mathbf{J}(x)=J_{m}(0) e^{-\frac{x}{\delta_{w}}} e^{-j \frac{x}{\delta_{w}}} e^{j \phi_{o}}=J_{m}(x) e^{j\left(\phi_{o}-\frac{x}{\delta_{w}}\right)}
$$

where the amplitude is

$$
J_{m}(x)=J_{m}(0) e^{-\frac{x}{\delta_{w}}} .
$$

The time-averaged point power density for sinusoidal waveforms is given by point Joule's law in phasor form:

$$
P_{D}(x)=\frac{1}{2} \operatorname{Re}\left(\mathbf{J} \cdot \mathbf{E}^{*}\right)=\frac{1}{2} \rho \mathbf{J} \cdot \mathbf{J}^{*}=\frac{1}{2} \rho|J(x)|^{2}=\frac{1}{2} \rho J_{m}^{2}(0) e^{-\frac{2 x}{\delta_{w}}},
$$

where $\delta_{w}$ is the skin depth. For periodic waveforms, the time-averaged real power dissipated in a conductor of volume $V$ and resistivity $\rho$ due to conversion of electromagnetic energy to thermal energy (heat) is given by Joule's law in phasor form:

$$
P_{D}=\frac{1}{2} \operatorname{Re} \iiint_{V} \mathbf{J} \cdot \mathbf{E}^{*} d V=\frac{1}{2} \iiint_{V} \rho \mathbf{J} \cdot \mathbf{J}^{*} d V=\frac{1}{2} \iiint_{V} \rho|J|^{2} d V,
$$

where $J$ and $E$ are the amplitudes of the current density and the electric field intensity, respectively. The time-averaged power loss density $P_{v}$ is defined as the total time-averaged power loss $P_{D}$ per unit volume,

$$
P_{v}=\frac{P_{D}}{V}
$$

where $V$ is the volume carrying the current.

Since $\mathbf{B}=\mu \mathbf{H}$, the point (local) magnetic energy density for sinusoidal waveforms is given by

$$
w_{m}(x)=\frac{1}{2} \mathbf{B} \cdot \mathbf{H}^{*}=\frac{1}{2} \mu \mathbf{H} \cdot \mathbf{H}^{*}=\frac{1}{2} \mu|H(x)|^{2} .
$$


The total magnetic energy stored in inductor $L$ is given by

$$
\begin{aligned}
W_{m}=\int i v d t=\int i L \frac{d i}{d t} d t & =L \int_{0}^{I_{m}} i d i=\frac{1}{2} L I_{m}^{2}=\frac{1}{2} \frac{N^{2}}{R}=\frac{F_{m}^{2}}{2 R}=\frac{1}{2} \frac{\mu N^{2} S}{l}\left(\frac{B l}{\mu N}\right)^{2} \\
& =\frac{1}{2} \iiint_{V} \mu|H(x)|^{2} d V,
\end{aligned}
$$

where $V$ is the volume of the interior of the inductor and $v=L d i / d t$. The magnetic energy density $w_{m}$ is defined as the magnetic energy $W_{m}$ per unit volume,

$$
w_{m}=\frac{W_{m}}{V} .
$$

The time-averaged local magnetic energy density is given by

$$
w_{m}(x)=\frac{1}{2} \mathbf{B} \cdot \mathbf{H}^{*}=\frac{1}{2} \mu \mathbf{H} \cdot \mathbf{H}^{*}=\frac{1}{2} \mu|H(x)|^{2}\left(\frac{\mathrm{J}}{\mathrm{m}^{3}}\right) .
$$

The total time-averaged magnetic energy is

$$
W_{m}=\frac{1}{2} \iiint_{V} \mathbf{B}(\mathbf{x}) \cdot \mathbf{H}^{*}(x) d V=\frac{1}{2} \iiint_{V} \mu|H(x)|^{2} d V(\mathbf{J}) .
$$

\subsection{Eddy Currents}

Figure 1.8 illustrates eddy current induced by a time-varying magnetic field. Eddy currents circulate in closed paths. In a conductor, the induced magnetic field may be caused by the conductor's own ac current or by the ac current of other adjacent conductors. According to Lenz's law, the magnetic field induces eddy currents, which generate magnetic field that opposes the original magnetic field. According to Ampère's law,

$$
\Delta \times \mathbf{H}=\mathbf{J}_{a}+\mathbf{J}_{e},
$$

where $\mathbf{J}_{a}$ is the applied current and $\mathbf{J}_{e}$ is the eddy current. When the applied current $\mathbf{J}_{a}$ is zero and the magnetic field is generated by adjacent conductors, we have

$$
\Delta \times \mathbf{H}=\mathbf{J}_{e} .
$$

The eddy-current density can be described by

$$
\mathbf{J}_{e}=\sigma \mathbf{E}=\frac{\mathbf{E}}{\rho} .
$$

For sinusoidal waveforms, the phasor of the eddy-current density is given by

$$
\mathbf{J}_{e}=-j \omega \sigma \mathbf{A},
$$

where $\mathbf{A}$ is the phasor of the magnetic vector potential.

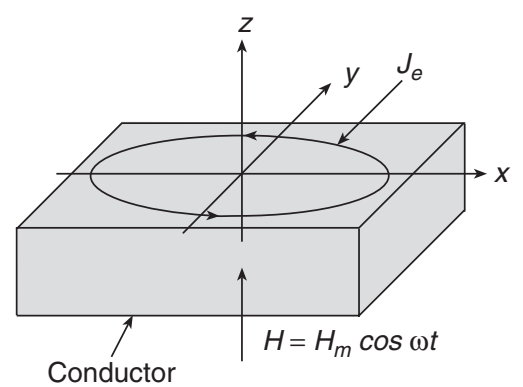

Figure 1.8 Eddy current. 


\subsection{Core Saturation}

For an inductor with a magnetic core of cross-sectional area $A_{c}$ and a saturation magnetic flux density $B_{s}$, the magnetic flux at which the magnetic core begins to saturate is

$$
\phi_{s}=A_{c} B_{s} .
$$

Hence, the magnetic flux linkage at which the magnetic core begins to saturate is given by

$$
\lambda_{s}=N \phi_{s}=N A_{c} B_{s}=L I_{m(\max )} .
$$

Thus,

$$
N_{\max } A_{c} B_{p k}=L I_{m(\max )},
$$

yielding the maximum number of turns

$$
N_{\max }=\frac{L I_{m(\max )}}{A_{c} B_{\max }} .
$$

According to (1.2), the magnetic field intensity $H$ is proportional to $\mathcal{F}=N i$. Therefore, there is a maximum amplitude of the inductor current $I_{m(\max )}$ at which the core saturates. Figure 1.9 shows plots of $B$ as a function of $H$ and $i$. Since

$$
B_{s}=\mu H_{s}=\mu \frac{N I_{m(\max )}}{l_{c}},
$$

to avoid core saturation, the ampere-turn limit is given by

$$
N_{\max } I_{m(\max )}=\frac{B_{s} l_{c}}{\mu_{r c} \mu_{0}}=B_{s} A_{c} \mathcal{R}=\frac{B_{s} l_{c}}{\mu_{r c} \mu_{0}} .
$$

From Faraday's law, $d \lambda=v_{L}(t) d t$. Hence, the general relationship between the inductor voltage and the flux linkage is given by

$$
\lambda(t)=\int_{0}^{t} v_{L}(t) d t+\lambda(0)=\frac{1}{\omega} \int_{0}^{\omega t} v_{L}(\omega t) d(\omega t)+\lambda(0) .
$$

For a transformer,

$$
\left(N_{1} i_{1}+N_{2} i_{2}+\cdots\right)_{\max } \leq B_{s} A_{c} R=\frac{B_{s} l_{c}}{\mu_{r c} \mu_{0}} .
$$

\subsubsection{Core Saturation for Sinusoidal Inductor Voltage}

Consider an inductor with a magnetic core of saturation flux density $B_{s}$. Figure 1.10 shows sinusoidal waveforms of the inductor voltage $v_{L}$ and the magnetic flux linkage $\lambda$. The dc components of these waveforms are assumed to be zero. The inductor voltage is given by

$$
v_{L}=V_{L m} \sin \omega t \text {. }
$$

The magnetic flux linkage is

$$
\begin{aligned}
\lambda(t) & =\frac{1}{\omega} \int_{0}^{\omega t} v_{L}(\omega t) d(\omega t)+\lambda(0)=\frac{1}{\omega} \int_{0}^{\omega t} V_{L m} \sin \omega t d(\omega t)+\lambda(0) \\
& =\frac{V_{L m}}{\omega}(1-\cos \omega t)+\lambda(0)=\frac{V_{L m}}{\omega}-\frac{V_{L m}}{\omega} \cos \omega t+\lambda(0) .
\end{aligned}
$$

Thus, the peak-to-peak value of the magnetic flux linkage is

$$
\Delta \lambda=\lambda(\pi)-\lambda(0)=\frac{2 V_{L m}}{\omega}=N \phi=N A_{c} B_{m}<N A_{c} B_{s} .
$$




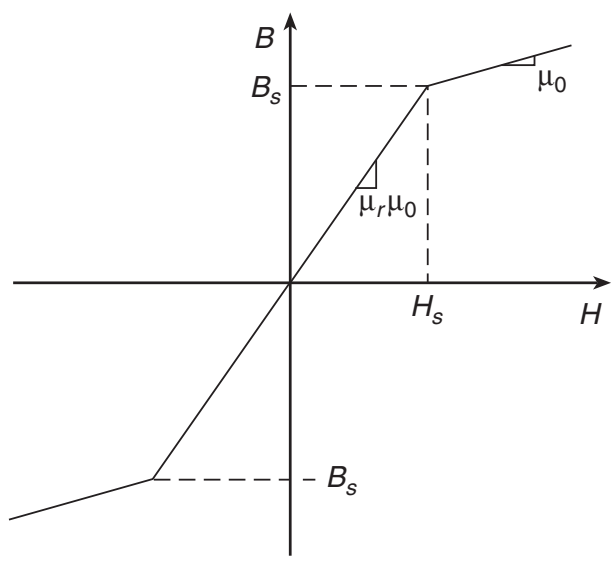

(a)

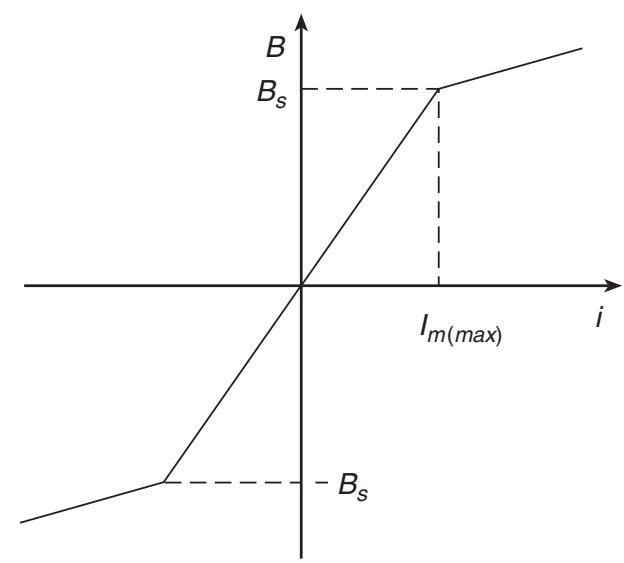

(b)

Figure 1.9 (a) Magnetic flux density $B$ as a function of magnetic field intensity $H$. (b) Magnetic flux density $B$ as a function of inductor current $i$ at a fixed number of turns $N$.

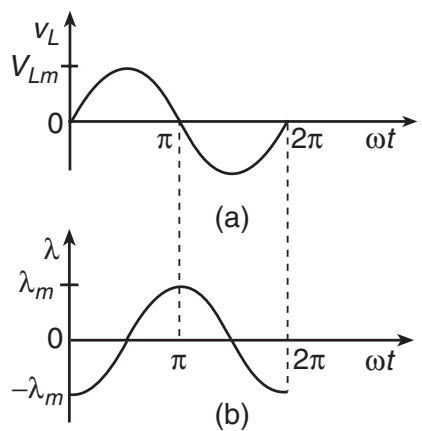

Figure 1.10 Waveforms of the inductor voltage and the magnetic flux linkage for sinusoidal inductor voltage. (a) Waveform of the inductor voltage $v_{L}$. (b) Waveform of the magnetic flux linkage $\lambda$. 
The initial value of the flux linkage is

$$
\lambda(0)=-\frac{\Delta \lambda}{2}=-\frac{V_{L m}}{\omega} .
$$

The steady-state waveform of the magnetic flux linkage is given by

$$
\lambda(t)=-\frac{V_{L m}}{\omega} \cos \omega t=-\lambda_{m} \cos \omega t,
$$

where the amplitude of the flux linkage is

$$
\lambda_{m}=\frac{V_{L m}}{\omega} .
$$

Thus, the amplitude of the magnetic flux linkage $\lambda_{m}$ increases as the frequency $f$ decreases. The minimum frequency $f_{\min }$ occurs when the amplitude of the magnetic flux linkage $\lambda_{m}$ reaches the saturation value $\lambda_{s}$ :

$$
\lambda_{m}=\lambda_{s}=\frac{V_{L m(\max )}}{\omega_{\min }} .
$$

The lowest frequency at which the inductor can operate without saturating the core is given by

$$
f_{\min }=\frac{V_{L m(\max )}}{2 \pi \lambda_{s}}=\frac{V_{L m(\max )}}{2 \pi N A_{c} B_{s}}=\frac{\sqrt{2} V_{L r m s(\max )}}{2 \pi N A_{c} B_{s}}=\frac{V_{L r m s(\max )}}{K_{f} N A_{c} B_{s}},
$$

where the waveform factor for a sinusoidal inductor voltage is

$$
K_{f}=\frac{2 \pi}{\sqrt{2}}=4.44 \text {. }
$$

The minimum frequency $f_{\min }$ decreases as $N$ increases, $A_{c}$ increases, $B_{s}$ increases, and $V_{L m(\max )}$ decreases. As the temperature increases, $B_{s}$ decreases. For ferrite cores, $B_{s}$ may decrease by a factor of 2 as $T$ increases from room temperature to $100^{\circ} \mathrm{C}$.

Another method to derive the minimum frequency is as follows. Assume that the initial condition is $\lambda(0)=-\lambda_{s}$. The magnetic flux linkage at core saturation is given by

$$
\lambda_{s}=\frac{1}{\omega_{\min }} \int_{0}^{\pi} v_{L} d(\omega t)+\lambda(0)=\frac{1}{\omega_{\min }} \int_{0}^{\pi} V_{L m} \sin \omega t d(\omega t)-\lambda_{s}=\frac{2 V_{L m}}{\omega_{\min }}-\lambda_{s},
$$

resulting in

$$
\lambda_{s}=\frac{\Delta \lambda_{\max }}{2}=\frac{V_{L m(\max )}}{\omega_{\min }} .
$$

Hence, the lowest frequency at which the inductor can operate without saturating the core is given by

$$
f_{\min }=\frac{V_{L m(\max )}}{2 \pi \lambda_{s}}=\frac{V_{L m(\max )}}{2 \pi N A_{c} B_{s}} .
$$

The maximum root mean square (rms) value of the sinusoidal voltage across an inductor is

$$
V_{\text {Lrms }(\max )}=\frac{\omega N A_{c} B_{s}}{\sqrt{2}}=\frac{2 \pi f N A_{c} B_{s}}{\sqrt{2}}=K_{f} f N A_{c} B_{s}=4.44 f N A_{c} B_{s} .
$$

\subsubsection{Core Saturation for Square Wave Inductor Voltage}

If the inductor voltage waveform is a square wave $\pm V$, the magnetic flux linkage is a symmetrical triangular wave, as shown in Figure 1.11. For the first half of the cycle,

$$
v_{L}=V, \quad \text { for } 0 \leq t \leq \frac{T}{2},
$$

and

$$
\lambda(t)=\int_{0}^{t} v_{L}(t) d t+\lambda(0)=\int_{0}^{t} V d t+\lambda(0)=V t+\lambda(0), \quad \text { for } \quad 0 \leq t \leq \frac{T}{2} .
$$




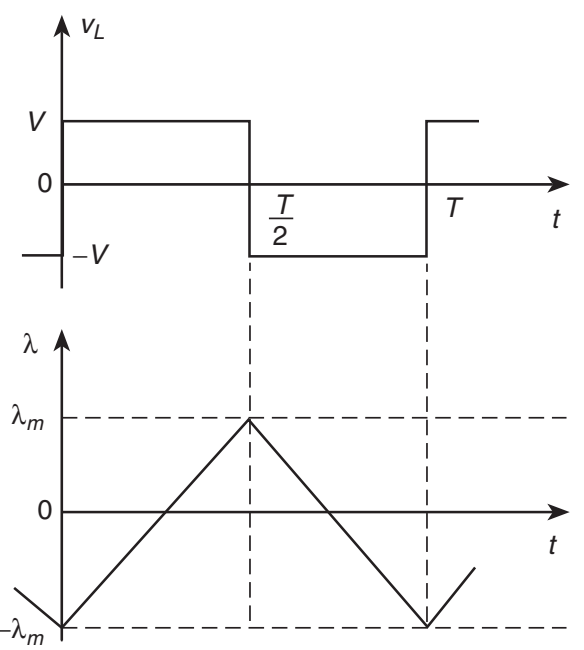

Figure 1.11 Waveforms of the inductor voltage and the magnetic flux linkage for a square wave inductor voltage.

The flux linkage at $t=T / 2$ is

$$
\lambda\left(\frac{T}{2}\right)=\frac{V T}{2}+\lambda(0) .
$$

For the second half of the cycle,

$$
v_{L}=-V, \text { for } \frac{T}{2} \leq t \leq T,
$$

and

$$
\begin{aligned}
\lambda(t) & =\int_{T / 2}^{t} v_{L}(t) d t+\lambda\left(\frac{T}{2}\right)=\int_{T / 2}^{t}(-V) d t+\lambda\left(\frac{T}{2}\right) \\
& =-V\left(t-\frac{T}{2}\right)+\lambda\left(\frac{T}{2}\right), \quad \text { for } \frac{T}{2} \leq t \leq T .
\end{aligned}
$$

Hence, the peak-to-peak value of the magnetic flux linkage is

$$
\begin{gathered}
\Delta \lambda=\lambda\left(\frac{T}{2}\right)-\lambda(0)=\frac{V T}{2}+\lambda(0)-\lambda(0)=\frac{V T}{2}=\frac{V}{2 f}, \\
-\lambda_{m}=\lambda(0)=-\frac{\Delta \lambda}{2}=-\frac{V T}{4}=-\frac{V}{4 f}
\end{gathered}
$$

and

$$
\lambda_{m}=\lambda\left(\frac{T}{2}\right)=\frac{\Delta \lambda}{2}=\frac{V T}{4}=\frac{V}{4 f} .
$$

The steady-state waveform of the magnetic linkage is

$$
\lambda(t)=V t-\frac{V}{4 f}, \quad \text { for } \quad 0 \leq t \leq \frac{T}{2},
$$

and

$$
\lambda(t)=-V\left(t-\frac{T}{2}\right)+\frac{V}{4 f}, \quad \text { for } \quad \frac{T}{2} \leq t \leq T .
$$

The rms value of the square wave inductor voltage is obtained as

$$
V_{r m s}=\sqrt{\frac{1}{T} \int_{0}^{T} v_{L}^{2} d t}=\sqrt{\frac{1}{T} \int_{0}^{T} V^{2} d t}=V .
$$


For core saturation,

$$
\lambda_{s}=\frac{\Delta \lambda_{m}}{2}=\frac{V_{\max }}{4 f_{\min }}=N \phi_{s}=N A_{c} B_{s},
$$

where $V_{\max }=V_{\text {Lrms }(\max )}$ for the square wave inductor current. The minimum frequency at which the core can be operated without saturation is given by

$$
f_{\min }=\frac{V_{\max }}{4 \lambda_{s}}=\frac{V_{\max }}{4 N A_{c} B_{s}}=\frac{V_{\max }}{K_{f} N A_{c} B_{s}}
$$

where the waveform coefficient of the square inductor voltage is

$$
K_{f}=4 \text {. }
$$

The maximum peak voltage of the square inductor voltage at the operating frequency $f$ is

$$
V_{r m s}=V_{\max }=4 f N A_{c} B_{s} .
$$

In general, the minimum core cross-sectional area is given by

where

$$
A_{c}=\frac{V_{\text {Lrms }}}{K_{f} f_{\text {min }} N B_{p k}}=\frac{V_{\text {Lrms }}}{K_{f} f_{\text {min }} N\left(B_{D C}+B_{m}\right)},
$$

$$
B_{p k}=B_{D C}+B_{m} \leq B_{s}, \text { for } T \leq T_{\max },
$$

and $K_{f}$ is the waveform coefficient of the inductor voltage. The peak value of the flux density $B_{p k}$ must be lower than $B_{s}$ at the maximum operating temperature $T_{\max }$ to avoid core saturation. The amplitude of the ac component of the flux density $B_{m}$ must be limited to avoid core saturation or to reduce core loss. As the amplitude of the ac component of the flux density $B_{m}$ increases, the core loss also increases.

The saturation flux density $B_{s}$ limits the maximum amplitude of the magnetic field intensity

$$
H_{s}=H_{m(\max )}=\frac{B_{s}}{\mu_{r c} \mu_{0}}=\frac{N I_{L m(\max )}}{l_{c}} .
$$

The maximum amplitude of the current in the winding at which the core saturates is

$$
I_{m(\max )}=\frac{l_{c} B_{s}}{N \mu_{r c} \mu_{0}} .
$$

As the amplitude of the inductor current $I_{L m}$ increases, the amplitude of the magnetic field $H_{m}$ also increases. To avoid core saturation,

$$
N I_{L m(\max )}<\frac{B_{s} l_{c}}{\mu_{r c} \mu_{0}} .
$$

When a core with an air gap is used, both amplitudes $H_{m}$ and $I_{L m}$ can be increased to

$$
H_{m(\max )}=\frac{B_{s}}{\mu_{r e} \mu_{0}}
$$

and

$$
I_{L m(\max )}=\frac{l_{c} B_{s}}{N \mu_{r e} \mu_{0}},
$$

where $\mu_{r e}$ is the core effective relative permeability.

\subsubsection{Core Saturation for Rectangular Wave Inductor Voltage}

Consider the situation where the inductor voltage waveform is a rectangular wave whose high level is $V_{H}$ and low level is $-V_{L}$, as depicted in Figure 1.12. The magnetic flux linkage is an asymmetrical triangular wave, as shown in Figure 1.11. For the first part of the cycle,

$$
v_{L}=V_{H}, \quad \text { for } 0 \leq t \leq D T,
$$




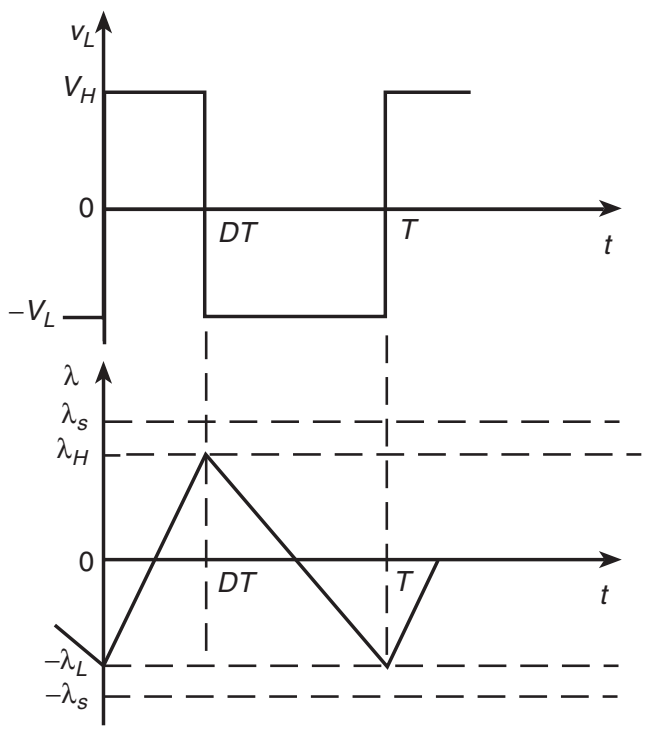

Figure 1.12 Waveforms of the inductor voltage and the magnetic flux linkage for a rectangular wave inductor voltage.

and

$$
\lambda(t)=\int_{0}^{t} v_{L}(t) d t+\lambda(0)=\int_{0}^{t} V_{H} d t+\lambda(0)=V_{H} t+\lambda(0), \quad \text { for } \quad 0 \leq t \leq D T,
$$

where $D$ is the duty cycle. The flux linkage at $t=D T$ is given by

$$
\lambda(D T)=V_{H} D T+\lambda(0) .
$$

For the second part of the cycle,

$$
v_{L}=-V_{L}, \quad \text { for } D T \leq t \leq T,
$$

and

$$
\begin{aligned}
\lambda(t) & =\int_{D T}^{t} v_{L}(t) d t+\lambda(D T)=\int_{D T}^{t}\left(-V_{L}\right) d t+\lambda(D T) \\
& =-V_{L}(t-D T)+\lambda(D T), \quad \text { for } D T \leq t \leq T .
\end{aligned}
$$

Hence, the peak-to-peak value of the magnetic flux linkage is

$$
\Delta \lambda=\lambda(D T)-\lambda(0)=V_{H} D T+\lambda(0)-\lambda(0)=V_{H} D T=\frac{D V_{H}}{f} .
$$

The rms value of the inductor voltage is

$$
V_{\text {Lrms }}=\sqrt{\frac{1}{T} \int_{0}^{T} v_{L}^{2} d t}=\sqrt{\frac{1}{T}\left(\int_{0}^{D T} V_{H}^{2} d t+\int_{D T}^{T} V_{L}^{2} d t\right)}=\sqrt{D V_{H}^{2}+(1-D) V_{L}^{2}} .
$$

Using the volt-second balance law, we obtain

$$
V_{H} D T=V_{L}(1-D) T
$$

yielding

$$
\frac{V_{L}}{V_{H}}=\frac{D}{1-D}
$$




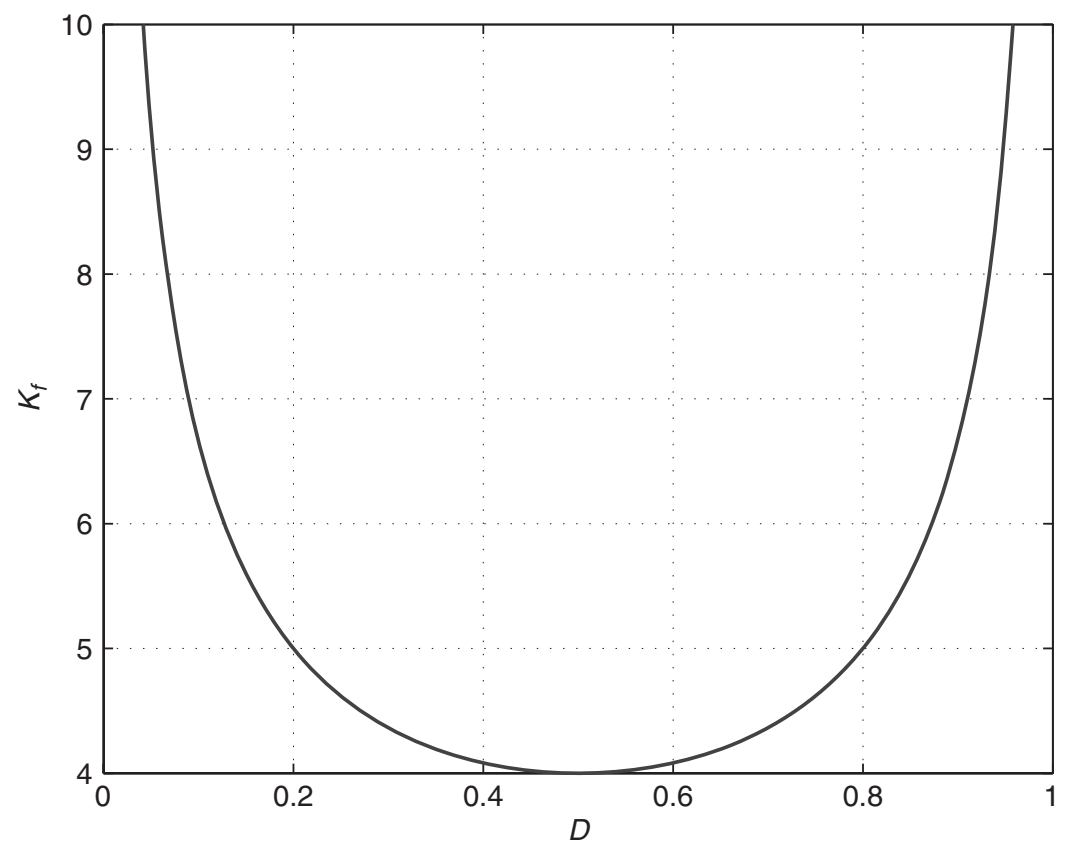

Figure 1.13 Waveform coefficient $K_{f}$ as a function of duty cycle $D$.

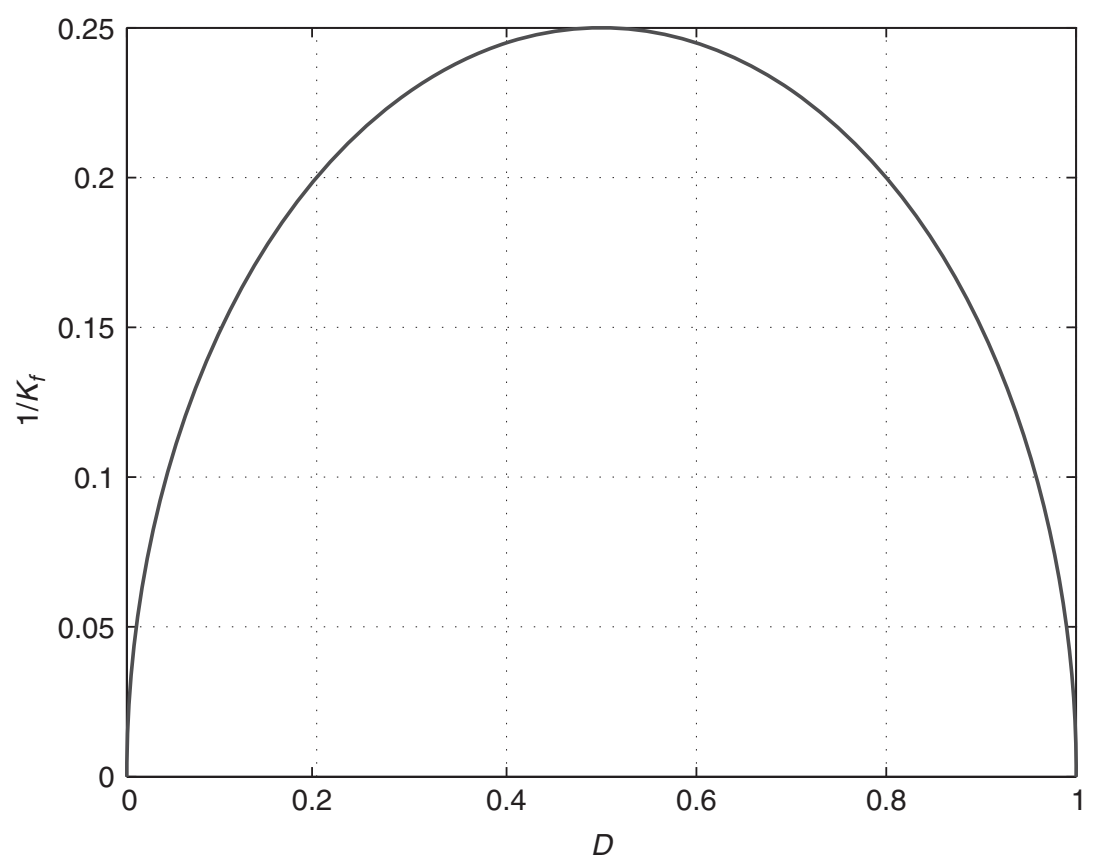

Figure 1.14 Coefficient $1 / K_{f}$ as a function of duty cycle $D$. 
Therefore,

$$
V_{\text {Lrms }}=V_{H} \sqrt{\frac{D}{1-D}}=V_{L} \sqrt{\frac{1-D}{D}} .
$$

The flux linkage at the beginning of core saturation is

$$
\lambda_{s}=\frac{\Delta \lambda_{\max }}{2}=\frac{D V_{H}}{2 f_{\min }}=N \phi_{s}=N A_{c} B_{s} .
$$

Hence, the minimum operating frequency is

$$
f_{\text {min }}=\frac{D V_{H}}{2 N A_{c} B_{s}}=\frac{V_{L r m s}}{N A_{c} B_{s}} \frac{\sqrt{D(1-D)}}{2}=\frac{V_{L r m s}}{K_{f} N A_{c} B_{s}},
$$

where the waveform coefficient is

$$
K_{f}=\frac{2}{\sqrt{D(1-D)}} .
$$

The minimum cross-sectional area is given by

$$
A_{c}=\frac{V_{\text {Lrms }}}{K_{\text {fmax }} f B_{s}} .
$$

Figure 1.13 shows a plot of $K_{f}$ as a function of the duty cycle $D$; like many of the figures in this book, it was created in MATLAB (see Appendix B). The minimum value of $K_{f}$ occurs at $D=0.5$. The lowest value of $f_{\min }$ occurs at $D=0.5$. Figure 1.14 depicts $1 / K_{f}$ as a function of $D$. The core cross-sectional area $A_{c}$ is proportional to $1 / K_{f}$. The maximum value of $A_{c}$ occurs at $D=0.5$.

\subsection{Volt-Second Balance}

For periodic waveforms in steady state,

$$
\lambda(T)-\lambda(0)=\int_{0}^{T} v_{L}(t) d t=0 .
$$

This equation is called a volt-second balance, which states that the area enclosed by the waveform $v_{L}$ above zero must be equal to the area enclosed by the waveform $v_{L}$ below zero for steady state. The volt-second balance can be expressed by

$$
\int_{0}^{t_{o}} v_{L}(t) d t=-\int_{t_{o}}^{T} v_{L}(t) d t
$$

\subsection{Inductance}

\subsubsection{Definitions of Inductance}

A coil is generally formed by winding a wire on a cylindrical former, called a bobbin. The inductance depends on (1) winding geometry, (2) core geometry, (3) permeability of the core material, and (4) frequency. There are several methods to determine the inductance.

\section{Magnetic Flux Linkage Method}

The inductance (or self-inductance) for linear inductors is defined as the ratio of the total magnetic flux linkage $\lambda$ to the time-varying (ac) current $i$, producing the flux linkage

$$
L=\frac{\lambda}{i}
$$




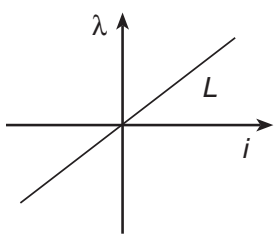

Figure 1.15 Magnetic flux linkage $\lambda$ as a function of current $i$ producing the flux linkage for linear inductors.

The inductance of a linear inductor is a proportionality constant in the expression $\lambda=L i$. In general, an ac current flowing through a conductor produces the magnetic linkage inside the conductor (an internal magnetic linkage) $\lambda_{\text {int }}$ and outside the conductor (an external magnetic linkage) $\lambda_{\text {ext }}$. Therefore, the inductance is defined as

$$
L=\frac{\lambda}{i}=\frac{\lambda_{\text {int }}+\lambda_{\text {ext }}}{i}
$$

A single conductor carrying an ac current $i$ is linked by its own magnetic flux. For linear inductors, the flux linkage $\lambda$ is proportional to current $i$, resulting in $\lambda=L i$. The inductance $L$ is the slope of the $\lambda-i$ characteristic, as illustrated in Figure 1.15. This characteristic is analogous to the resistor characteristic $v=R i$ or the capacitor characteristic $Q=C v$. A circuit that is designed to have a self-inductance is called an inductor. An inductor has a self-inductance of $1 \mathrm{H}$ if a current of $1 \mathrm{~A}$ produces a flux linkage of $1 \mathrm{~V} \cdot \mathrm{s}$ (or $1 \mathrm{~Wb} \cdot$ turn).

A change in the current flowing through the inductor produces an induced electromotive force, called an electromotance, or voltage

$$
\oint_{C} \mathbf{E} \cdot d \mathbf{l}=\frac{\lambda}{d t}=L \frac{d i}{d t} .
$$

An inductor has a self-inductance of $1 \mathrm{H}$ if the current flowing through it changes at a rate of $1 \mathrm{~A} / \mathrm{s}$ when the voltage difference between its terminals is $1 \mathrm{~V}$. The inductance $L$ is a function of the number of turns $N$, core permeability $\mu_{r c}$, core geometry, and frequency $f$.

The inductance can be defined as

$$
L=\frac{\lambda}{i}=\frac{1}{i} \iint_{S} \mathbf{B} \cdot d \mathbf{S}
$$

The magnetic field produced by a current-carrying conductor links itself. The associated inductance is called self-inductance. In some cases, the magnetic flux links only a part of the current and the inductance is defined as

$$
L=\frac{1}{i} \iint_{S} \frac{i_{\text {enclosed }}}{i} d \phi .
$$

The total inductance of a conductor is made up of two components: an external inductance $L_{e x t}$ and an internal inductance $L_{\text {int }}$ :

$$
L=L_{\text {ext }}+L_{\text {int }} .
$$

The external inductance $L_{\text {ext }}$ is due to the magnetic energy stored in the magnetic field outside the conductor. This inductance is usually independent of frequency. The internal inductance $L_{\text {int }}$ is due to the magnetic energy stored in the internal magnetic field inside the conductor. This inductance depends on frequency because the magnetic field intensity $H$ distribution inside the conductor is a function of frequency due to the skin effect. The internal inductance usually decreases with frequency.

The voltage across the inductance is

$$
v_{L}=\frac{d \lambda}{d t}=N \frac{d \phi}{d t}=N \frac{d \phi}{d i_{L}} \frac{d i_{L}}{d t}=L \frac{d i_{L}}{d t} .
$$

The self-inductance $L$ relates the voltage induced in an inductor $v_{L}$ to the time-varying current $i_{L}$ flowing through the same inductor. 


\section{Reluctance Method}

The inductance of an inductor can be determined using the core reluctance $\mathcal{R}$ or the core permeance $\mathcal{P}$ :

$$
L=\frac{N^{2}}{\mathcal{R}}=\mathcal{P} N^{2}=\frac{\mu_{r c} \mu_{0} A_{c} N^{2}}{l_{c}} .
$$

If $N=1, L=\mathcal{P}=1 / \mathcal{R}$.

\section{Magnetic Energy Method}

Inductance may be equivalently defined using magnetic energy,

$$
W_{m}=\frac{1}{2} L I_{m}^{2}=\frac{1}{2} \int_{V}\left(\mathbf{B} \cdot \mathbf{H}^{*}\right) d V,
$$

yielding the inductance

$$
L=\frac{2 W_{m}}{I_{m}^{2}}=\frac{1}{I_{m}^{2}} \int_{V}\left(\mathbf{B} \cdot \mathbf{H}^{*}\right) d V,
$$

where $I_{m}$ is the amplitude of the current flowing in the closed path and $W_{m}$ is the energy stored in the magnetic field produced by the current flowing through the inductor,

$$
W_{m}=\frac{1}{2 \mu} \iiint_{V} B^{2} d V .
$$

\section{Small-Signal Inductance}

The small-signal (or incremental) inductance of a nonlinear inductor is defined as the ratio of the infinitesimal change in the flux linkage to the infinitesimal change in the current producing it at a given operating point $Q\left(I_{D C}, \lambda_{D C}\right)$ :

$$
L=\left.\frac{d \lambda}{d i}\right|_{Q} .
$$

Inductors with ferrous cores are nonlinear because the permeability depends on the applied magnetic field $H$. Figure 1.16 shows a plot of the magnetic flux linkage $\lambda$ as a function of current $i$ for nonlinear inductors. At low values of current, the core is not saturated and the relative permeability is high, resulting in a high slope of the $\lambda-i$ curve and a large inductance $L_{1}$. When the core saturates, the

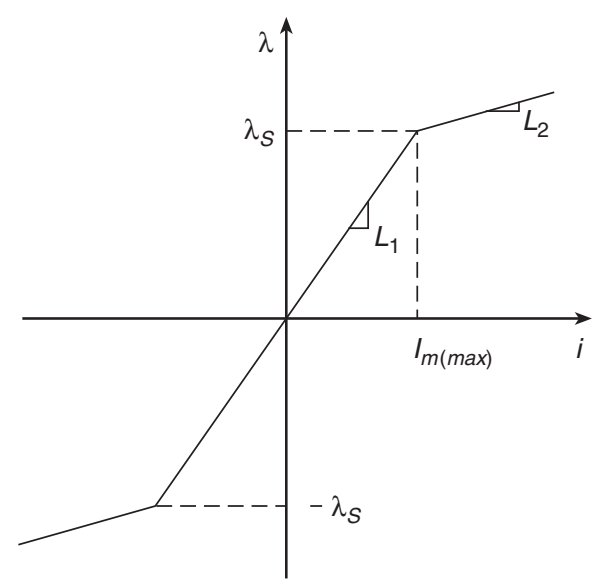

Figure 1.16 Magnetic flux linkage $\lambda$ as a function of current $i$ producing the flux linkage for nonlinear inductors. 


\section{8}

relative permeability $\mu_{r c}$ becomes equal to 1 , the slope of the $\lambda-i$ curve decreases and the inductance decreases to a lower value $L_{2}$.

\section{Vector Magnetic Potential Method}

The inductance can be determined using the vector magnetic potential $\mathbf{A}$ :

$$
L=\frac{1}{I^{2}} \iiint_{V} \mathbf{A} \cdot \mathbf{J} d V .
$$

The vector magnetic potential is given by

$$
\mathbf{A}(\mathbf{r})=\frac{\mu}{4 \pi} \iiint_{V} \frac{\mathbf{J}(\mathbf{r})}{R} d V .
$$

Hence, the inductance is given by

$$
L=\frac{1}{I^{2}} \iiint_{V}\left[\frac{\mu}{4 \pi} \iiint_{V} \frac{\mathbf{J}(\mathbf{r})}{R} d V\right] \cdot \mathbf{J}(\mathbf{r}) d V .
$$

\section{Example 1.2}

An inductor is wound on a CC core (see Figure 2.9) whose cross-sectional area is $2 \mathrm{~cm} \times 2 \mathrm{~cm}$, $l_{c}=16 \mathrm{~cm}$, the core window is $3 \mathrm{~cm} \times 3 \mathrm{~cm}$, and $\mu_{r c}=100$. The core has no air gap. There is a magnetic flux in the core $\phi_{c}$ and a leakage flux $\phi_{l}$ in the air. The inductor has 10 turns. Estimate the inductance using the reluctance method.

Solution: The total magnetic flux is

$$
\phi=\phi_{c}+\phi_{l}
$$

The inductance is

$$
L=\frac{N \phi}{i}=N^{2}\left(\frac{1}{R_{c}}+\frac{1}{R_{l}}\right)=N^{2}\left(\frac{\mu_{r c} \mu_{0} A_{c}}{l_{c}}+\frac{\mu_{0} A_{l}}{l_{l}}\right) .
$$

Let $l_{l}=l_{c} / 2$ and $A_{l}=4 A_{c}$. In this case, the inductance is given by

$$
\begin{aligned}
L & =N^{2}\left(\frac{\mu_{r c} \mu_{0} A_{c}}{l_{c}}+\frac{8 \mu_{0} A_{c}}{l_{c}}\right)=N^{2}\left(\frac{\mu_{0} A_{c}}{l_{c}}\right)\left(\mu_{r c}+8\right) \\
& =10^{2}\left(\frac{4 \pi \times 10^{-7} \times 4 \times 10^{-4}}{16 \times 10^{-2}}\right)(100+8)=33.929 \mu \mathrm{H} .
\end{aligned}
$$

The inductance is increased by $8 \%$ due to the leakage magnetic flux.

\subsubsection{Inductance of Solenoid}

Neglecting the end effects, the magnetic flux density inside a long solenoid is uniform and it is given by

$$
B=\frac{\mu N I}{l_{c}} .
$$

The magnetic flux inside is

$$
\phi=A_{c} B=\frac{\mu N I A_{c}}{l_{c}}=\frac{\pi \mu N I r^{2}}{l_{c}} .
$$


The flux linkage is

$$
\lambda=N \phi=\frac{\mu N^{2} I A_{c}}{l_{c}}=\frac{\pi \mu N^{2} I r^{2}}{l_{c}} .
$$

The inductance of a long solenoid (theoretically infinitely long) with a core and without an air gap at low frequencies is

$$
L_{\infty}=\frac{\lambda}{I}=\frac{\mu_{r c} \mu_{0} A_{c} N^{2}}{l_{c}}=\frac{\pi \mu_{r c} \mu_{0} r^{2} N^{2}}{l_{c}}=\frac{N^{2}}{l_{c} /\left(\mu A_{c}\right)}=\frac{N^{2}}{\mathcal{R}}
$$

where $A_{c}=\pi r^{2}$ is the core cross-sectional area, $r$ is the mean coil radius, $l_{c}$ is the mean core length, $\mu_{r c}$ is the relative permeability of the core, and $N$ is the total number of turns. The inductance $L$ is proportional to the square of the number of turns $N^{2}$ and the cross-sectional area $A_{c}$, and is inversely proportional to its length $l_{c}$. More precisely, the inductance $L$ is proportional to the ratio of the core cross-sectional area to the magnetic path length $A_{c} / l_{c}$.

The inductance of a short solenoid is smaller than that of an infinitely long round solenoid. As $r / l_{c}$ increases, $L / L_{\infty}$ decreases, where $L_{\infty}$ is the inductance of an infinitely long solenoid. For example, $K=L / L_{\infty}=0.85$ for $r / l_{c}=0.2, K=0.74$ for $r / l_{c}=0.4, K=0.53$ for $r / l_{c}=1, K=0.2$ for $r / l_{c}=5$, and $K=0.12$ for $r / l_{c}=10$. A first-order approximation is

$$
K=\frac{L}{L_{\infty}} \approx \frac{1}{1+0.9 \frac{r}{l_{c}}},
$$

resulting in $L \approx K L_{\infty}=L_{\infty} /\left(1+0.9 r / l_{c}\right)$. The inductance of a round single-layer solenoid of a finite length $l_{c}$ can be approximated by Wheeler's formula [38], which is correct to within $1 \%$ for $r / l_{c}<1.25\left(\right.$ or $\left.l_{c} /(2 r)>0.4\right)$ :

$$
\begin{aligned}
L=\frac{L_{\infty}}{1+0.9 \frac{r}{l_{c}}} & =\frac{\mu_{r c} \mu_{0} A_{c} N^{2}}{l_{c}\left(1+0.9 \frac{r}{l_{c}}\right)}=\frac{\pi \mu_{r c} \mu_{0} r^{2} N^{2}}{l_{c}\left(1+0.9 \frac{r}{l_{c}}\right)}=\frac{\pi \mu_{r c} \mu_{0} r^{2} N^{2}}{l_{c}+0.9 r} \\
& =\frac{0.4 \pi^{2} \mu_{r c} r^{2} N^{2}}{l_{c}+0.9 r}(\mu \mathrm{H}), \quad \text { for } \frac{r}{l_{c}}<1.25 .
\end{aligned}
$$

Figure 1.17 shows a plot of $L / L_{\infty}$ as a function of $r / l_{c}$. As the ratio of the external diameter to the internal diameter decreases, the inductance also decreases.

The inductance of a multi-layer solenoid is given by

$$
L=\frac{0.8 \mu \pi r^{2} N^{2}}{l_{c}+0.9 r+b}
$$

where $b$ is the thickness of all layers (or coil build) and $r$ is the average radius of the winding.

A more accurate equation for the inductance of a multi-layer inductor is

$$
L=\frac{\mu \pi r^{2} N^{2}}{l_{c}} \frac{1}{1+0.9 \frac{r}{l_{c}}+0.32 \frac{b}{r}+0.84 \frac{b}{l_{c}}} .
$$

The inductance predicted by this equation is within $2 \%$ of the exact value.

\section{Example 1.3}

An air core solenoid has $N=20, l_{c}=15 \mathrm{~cm}$, and $r=3 \mathrm{~cm}$. Find the inductance.

Solution: The inductance of the solenoid is

$$
L=\frac{\pi \mu_{r c} \mu_{0} r^{2} N^{2}}{l_{c}\left(1+0.9 \frac{r}{l_{c}}\right)}=\frac{\pi \times 1 \times 4 \pi \times 10^{-7} \times\left(3 \times 10^{-2}\right)^{2} \times 20^{2}}{15 \times 10^{-2}\left(1+0.9 \times \frac{3 \times 10^{-2}}{15 \times 10^{-2}}\right)}=8.0295 \mu \mathrm{H} .
$$

Note that the inductance calculated in this example is about $15 \%$ less than the inductance calculated for a very long inductor because $K=0.8475$. 


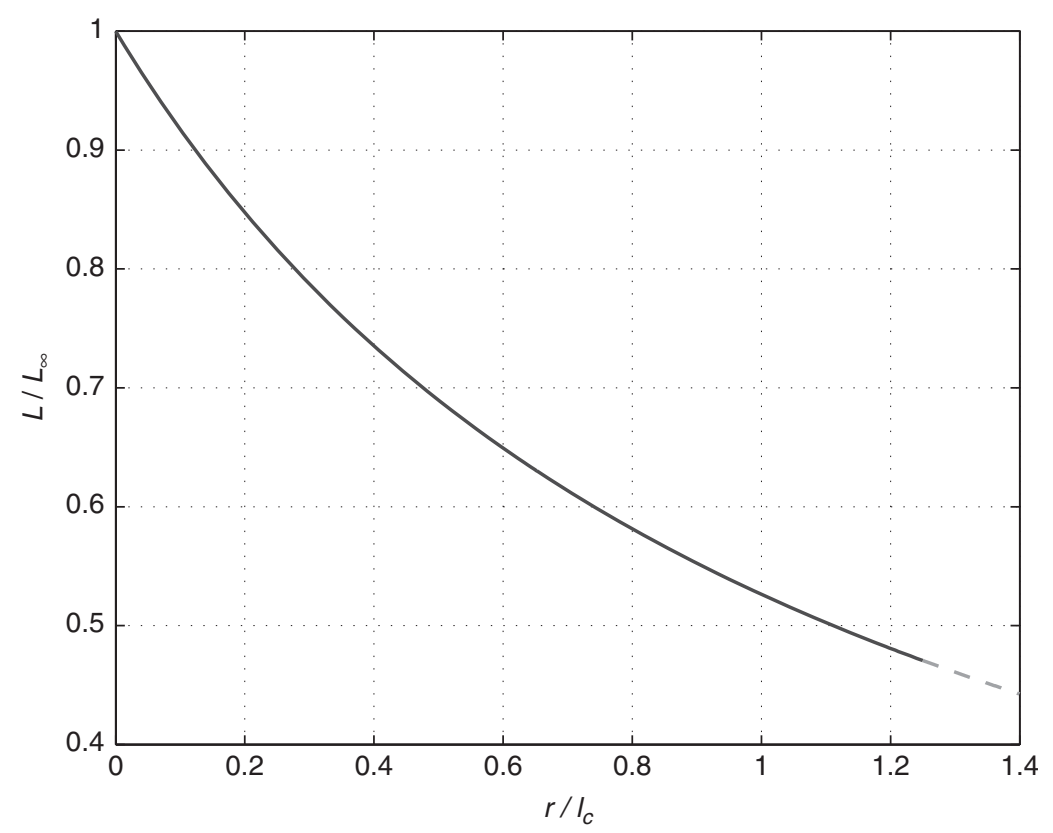

Figure 1.17 Plot of $L / L_{\infty}$ as a function of $r / l_{c}$.

\subsubsection{Inductance of Inductor with Toroidal Core}

An idealized toroid can be thought as a finite-length solenoid bent around to close on itself to form a doughnut shape. A toroidal inductor with a rectangular cross section is shown in Figure 1.18. The dimensions of the magnetic core are: $a$ is the inner radius, $b$ is the outer radius, and $h$ is the toroid height. The toroid is symmetrical about its axis. Thus,

$$
d l=r d \varphi .
$$

Applying Ampère's law,

$$
\oint_{C} \mathbf{B} \cdot d \mathbf{l}=\int_{0}^{2 \pi} B r d \varphi=B r \int_{0}^{2 \pi} d \varphi=2 \pi r B .
$$
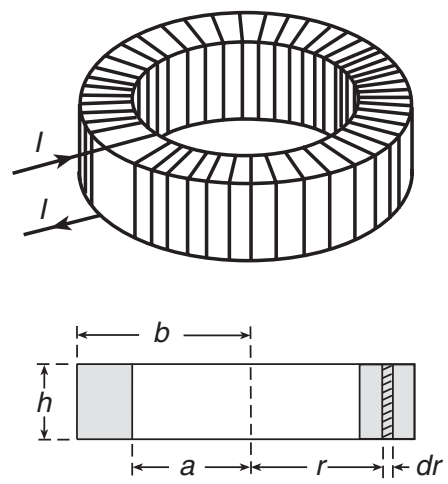

Figure 1.18 Toroidal inductor. 
Since the path of integration encircles a total current $N I$, we obtain

$$
2 \pi r B=\mu N I .
$$

Hence, the magnetic flux density inside the toroidal core is given by

$$
B(r)=\frac{\mu N I}{2 \pi r}, \text { for } a \leq r \leq b .
$$

Since $d S=h d r$, the magnetic flux inside the toroidal core is

$$
\begin{aligned}
\phi=\iint_{S} B(r) d S=\int_{a}^{b} \int_{0}^{h}\left(\frac{\mu N I}{2 \pi r}\right)(h d r) & =\frac{\mu N I h}{2 \pi} \int_{a}^{b} \frac{d r}{r}=\int_{S}\left(\frac{\mu N I}{2 \pi r}\right)(h d r) \\
& =\frac{\mu N I h}{2 \pi} \ln \left(\frac{b}{a}\right)
\end{aligned}
$$

where $S$ is the surface bounded by the path $C$. The flux linkage of the toroidal inductor is

$$
\lambda=N \phi=\frac{\mu h N^{2} I}{2 \pi} \ln \left(\frac{b}{a}\right)
$$

resulting in the inductance of a toroidal coil

$$
L=\frac{\lambda}{I}=\frac{\mu_{r c} \mu_{0} h N^{2}}{2 \pi} \ln \left(\frac{b}{a}\right) .
$$

The inductance of a toroidal coil with a round cross section can be described by the expression for the inductance of a long solenoid

$$
L=\frac{\mu_{r c} \mu_{0} A_{c} N^{2}}{l_{c}}=\frac{\mu_{r c} \mu_{0} A_{c} N^{2}}{2 \pi R}
$$

where $R=(a+b) / 2$ is the mean radius of the core, $l_{c}=2 \pi R=\pi(a+b)$, and $A_{c}=\pi(b-a)^{2} / 4$ is the cross-sectional area of the core. Hence,

$$
L=\frac{\mu_{r c} \mu_{0} N^{2}(b-a)^{2}}{4(a+b)} .
$$

\section{Example 1.4}

An inductor is wound on a toroidal core where $\mu_{r c}=150, h=1 \mathrm{~cm}, a=4 \mathrm{~cm}$, and $b=5 \mathrm{~cm}$. The inductor has 20 turns. Find the inductance.

Solution: The inductance is

$$
L=\frac{\mu_{r c} \mu_{0} h N^{2}}{2 \pi} \ln \left(\frac{b}{a}\right)=\frac{150 \times 4 \pi \times 10^{-7} \times 10^{-2} \times 20^{2}}{2 \pi} \ln \left(\frac{5}{4}\right)=26.777 \mu \mathrm{H} .
$$

\subsubsection{Inductance of Inductor with Pot Core}

The geometry of an inductor with a pot core is very complex and the inductance of these inductors can be determined only approximately. The core cross-sectional area of the pot core is approximately equal to the cross-sectional area of the center post,

$$
A_{c}=\frac{\pi d^{2}}{4}
$$


where $d$ is the diameter of the center post. The average diameter of the mean magnetic path is given by

$$
D_{a v}=\frac{D_{i}+D_{o}}{2}
$$

where $D_{i}$ is the inner diameter of the outer core area and $D_{o}$ is the outer diameter of the outer core area. The mean magnetic path length is given by

$$
l_{c}=2 D_{a v}+4 H=D_{i}+D_{o}+4 H
$$

where $H$ is the height of the core halve. The inductance of an inductor with a pot core can be approximated by

$$
L=\frac{\mu_{r c} \mu_{0} A_{c} N^{2}}{l_{c}}=\frac{\pi \mu_{r c} \mu_{0} d^{2} N^{2}}{4\left(D_{i}+D_{o}+4 H\right)} .
$$

\subsubsection{Air Gap}

The overall reluctance $\mathcal{R}$ can be controlled by an air gap in the core. Therefore, the magnetic flux $\phi$, flux density $B$, and inductance $L$ can be controlled by the length of the air gap $l_{g}$. Air gaps can be bulk or distributed. In a gapped core, a small section of the magnetic flux path is replaced by a nonmagnetic medium, such as air or nylon. It is often filled with a spacer. The air gap length $l_{g}$ is usually twice the spacer thickness. Some cores have pre-fabricated air gaps. Standard values of $l_{g}$ are $0.5,0.6,0.7, \ldots, 5 \mathrm{~mm}$. Adding an air gap in a core is equivalent to adding a large gap reluctance in series with the core reluctance (i.e., a series reluctor). As a result, the magnitude of the magnetic flux $\phi_{m}$ at a fixed value of $N I_{m}$ is reduced. This effect is analogous to adding a series resistor in an electric circuit to reduce the magnitude of the current at a fixed source voltage.

Figure 1.19(a) illustrates an inductor whose core has an air gap. An equivalent magnetic circuit of an inductor with an air gap is shown in Figure 1.19(b). The inductance of a coil with a magnetic core having an air gap at low frequencies is expressed as

$$
\begin{aligned}
L & =\frac{N^{2}}{\mathcal{R}_{g}+\mathcal{R}_{c}}=\frac{N^{2}}{\frac{l_{g}}{\mu_{0} A_{c}}+\frac{l_{c}}{\mu_{r c} \mu_{0} A_{c}}}=\frac{\mu_{r c} \mu_{0} A_{c} N^{2}}{l_{c}+\mu_{r c} l_{g}}=\frac{\mu_{0} A_{c} N^{2}}{l_{g}+\frac{l_{c}}{\mu_{r c}}} \\
& =\frac{\mu_{r c} \mu_{0} A_{c} N^{2}}{l_{c}\left(1+\frac{\mu_{r c} l_{g}}{l_{c}}\right)}=\frac{\mu_{r c} \mu_{0} A_{c} N^{2}}{l_{c} F_{g}},
\end{aligned}
$$

where the reluctance of the air gap is

$$
\mathcal{R}_{g}=\frac{l_{g}}{\mu_{0} A_{c}}
$$

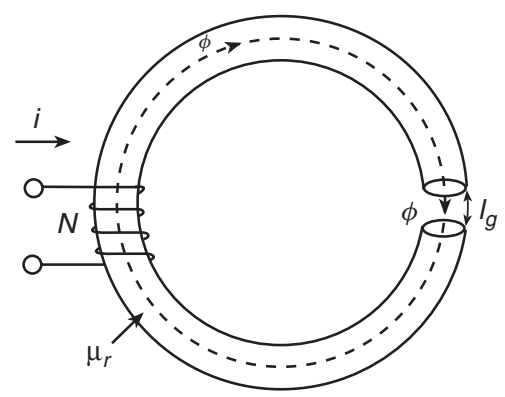

(a)

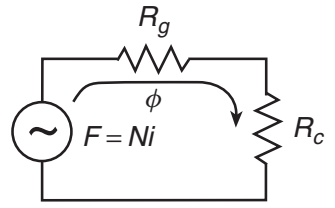

(b)

Figure 1.19 Inductor with an air gap. (a) Inductor. (b) Magnetic circuit of an inductor with an air gap. 
the reluctance of the core is

$$
\mathcal{R}_{c}=\frac{l_{c}-l_{g}}{\mu_{r c} \mu_{0} A_{c}} \approx \frac{l_{c}}{\mu_{r c} \mu_{0} A_{c}},
$$

the overall reluctance is

$$
\mathcal{R}=\mathcal{R}_{c}+\mathcal{R}_{g}=\frac{l_{c}}{\mu_{r c} \mu_{0} A_{c}}+\frac{l_{g}}{\mu_{0} A_{c}}=\frac{l_{c}}{\mu_{r c} \mu_{0} A_{c}}\left(1+\frac{\mu_{r c} l_{g}}{l_{c}}\right)=F_{g} \mathcal{R}_{c},
$$

the air gap factor is

$$
F_{g}=\frac{\mathcal{R}}{\mathcal{R}_{c}}=\frac{\mathcal{R}_{c}+\mathcal{R}_{g}}{\mathcal{R}_{c}}=1+\frac{\mathcal{R}_{g}}{\mathcal{R}_{c}}=1+\frac{\mu_{r c} l_{g}}{l_{c}},
$$

and the effective relative permeability of a core with an air gap is

$$
\mu_{r e}=\frac{\mu_{r c}}{1+\frac{\mu_{r c} l_{g}}{l_{c}}}=\frac{\mu_{r c}}{F_{g}} .
$$

The air gap causes a considerable decrease in the effective relative permeability. However, it produces a more stable effective permeability and reluctance, resulting in a more predictable and stable inductance. For example, inductors used in resonant circuits should be predictable and stable. Usually, at least $95 \%$ of the inductance comes from the air gap. The length of the air gap is given by

$$
l_{g}=\frac{\mu_{0} A_{c} N^{2}}{L}-\frac{l_{c}}{\mu_{r c}} .
$$

The number of turns of an inductor whose core has an air gap is given by

$$
N=\sqrt{\frac{L\left(l_{g}+\frac{l_{c}}{\mu_{r c}}\right)}{\mu_{0} A_{c}} .}
$$

For $l_{g} \gg l_{c} / \mu_{r c}, \mathcal{R}_{g} \gg \mathcal{R}_{c}$, and

$$
L \approx \frac{\mu_{0} A_{c} N^{2}}{l_{g}}=\frac{N^{2}}{\mathcal{R}_{g}} .
$$

Therefore, the inductance of an inductor with a core air gap is a function of the air gap length $l_{g}$ and is almost independent of the core relative permeability $\mu_{r c}$. The number of turns is

$$
N \approx \sqrt{\frac{L l_{g}}{\mu_{0} A_{c}}}, \text { for } l_{g} \gg \frac{l_{c}}{\mu_{r c}} .
$$

The core permeability varies with temperature and flux level. Inductors that carry dc currents and have dc magnetic flux require long air gaps to avoid saturation.

The relationships for the inductor with an air gap can be written as

$$
\mathcal{F}=N i=H_{c} l_{c}+H_{g} l_{g}=\frac{B_{c} l_{c}}{\mu_{r c} \mu_{0}}+\frac{B_{g} l_{g}}{\mu_{0}}=\frac{\phi_{c} l_{c}}{A_{c} \mu_{r c} \mu_{0}}+\frac{\phi_{g} l_{g}}{A_{a} \mu_{0}}=\mathcal{R}_{c} \phi_{c}+\mathcal{R}_{g} \phi_{g} .
$$

For $\mathcal{R}_{g} \gg \mathcal{R}_{c}, \phi \approx N i / R_{g}$. Neglecting the flux fringing effect, $B_{g}=B_{c}$. Hence,

$$
N i=B_{c}\left(\frac{l_{c}}{\mu_{r c} \mu_{0}}+\frac{l_{g}}{\mu_{0}}\right) .
$$

The magnetic flux density in the core with an air gap is given by

$$
B_{c}=\frac{\mu_{0} N i}{l_{g}+\frac{l_{c}}{\mu_{r c}}} .
$$


Hence, the maximum flux density in the core with an air gap, which is caused by the dc component of the inductor current $I_{L}$ and the amplitude of the ac component of the inductor current $I_{m}$, is expressed by

$$
B_{c(p k)}=B_{D C}+B_{m}=\frac{\mu_{0} N\left(I_{L}+I_{m}\right)}{l_{g}+\frac{l_{c}}{\mu_{r c}}} \leq B_{s}, \quad \text { for } T \leq T_{\max } .
$$

The magnetic flux density and the magnetic field intensity in the core are

$$
B_{c}=\frac{\phi_{c}}{A_{c}}
$$

and

$$
H_{c}=\frac{B_{c}}{\mu_{r c} \mu_{0}} .
$$

Assuming a uniform magnetic flux density in the air gap and neglecting the fringing effect, the magnetic flux, magnetic flux density, and magnetic field intensity in the air gap are

$$
\begin{aligned}
& \phi_{g}=\phi_{c}=A_{c} B_{c}=A_{g} B_{g}, \\
& B_{g}=\frac{A_{c}}{A_{g}} B_{c} \approx B_{c},
\end{aligned}
$$

and

$$
H_{g}=\frac{B_{g}}{\mu_{0}}=\frac{B_{c}}{\mu_{0}}=\mu_{r c} H_{c} .
$$

Gap losses consist of winding loss, core loss, and hardware loss (e.g., power loss in clamps or bolts).

The maximum MMF is

$$
\mathcal{F}_{\text {max }}=N_{\text {max }} I_{\text {Lmax }}=\phi\left(\mathcal{R}_{g}+\mathcal{R}_{c}\right)=B_{p k} A_{c}\left(\mathcal{R}_{g}+\mathcal{R}_{c}\right) \approx B_{p k} A_{c} \mathcal{R}_{g}=\frac{B_{p k} l_{g}}{\mu_{0}},
$$

where $\mathcal{R}_{g}=l_{g} /\left(\mu_{0} A_{c}\right)$. To avoid core saturation, the maximum number of turns is given by

$$
N_{\max }=\frac{B_{p k} l_{c}}{\mu_{0} I_{\text {Lmax }}} .
$$

As the air gap length $l_{c}$ increases, $N I_{m}$ can be increased and the core loss decreases. However, the number of turns $N$ must be incressed to achieve a specified inductance $L$. Increasing the number of turns increases the winding loss. In addition, the leakage inductance increases and the air gap radiates a larger amount of electromagnetic interference (EMI).

The behavior of an inductor with an air gap is similar to an amplifier with negative feedback,

$$
A_{f}=\frac{A}{1+\beta A}=\frac{\mu_{r c}}{1+\mu_{r c} \frac{l_{g}}{l_{c}}} .
$$

Thus, $\mu_{r c}$ is analogous to $A$ and $l_{g} / l_{c}$ is analogous to $\beta$.

\section{Example 1.5}

A PQ4220 Magnetics core [48] has $\mu_{r c}=2500, l_{c}=4.63 \mathrm{~cm}$, and $A_{c}=1.19 \mathrm{~cm}^{2}$. The inductor wound on this core has $N=10$ turns. The required inductance should be $L=55.6 \mu \mathrm{H}$. Find the length of the air gap $l_{g}$.

Solution: The length of the air gap in the core is

$$
\begin{aligned}
l_{g}=\frac{\mu_{0} A_{c} N^{2}}{L}-\frac{l_{c}}{\mu_{r c}} & =\frac{4 \pi \times 10^{-7} \times 1.19 \times 10^{-4} \times 10^{2}}{55.6 \times 10^{-6}}-\frac{4.63 \times 10^{-2}}{2500} \\
& =(0.2689564-0.01852) \times 10^{-3}=0.2504 \mathrm{~mm} .
\end{aligned}
$$




\subsubsection{Fringing Flux}

A fringing flux is present around the air gap whenever the core is excited, as shown in Figure 1.20. Figure 1.21 depicts fringing flux in an inductor with an EE core (see Figure 2.14) and air gap. The magnetic flux lines bulge outward because the magnetic lines repel each other when passing through nonmagnetic material. As a result, the cross-sectional area of the magnetic field is increased and the flux density is decreased. Typically, $10 \%$ is added to the air gap cross-sectional area. This effect is called the fringing flux. The percentage of the fringing flux in the total magnetic flux increases as the air-gap length $l_{g}$ increases. The maximum increase in the radius of the magnetic flux due to the fringing effect is approximately equal to the length of the air gap length $l_{g}$. Figure 1.22 shows a magnetic equivalent circuit for the inductor with an air gap and fringing flux. The fringing permeance is shunting the gap permeance.

Due to the continuity of magnetic flux, the magnetic flux in the core $\phi_{c}$ is equal to the sum of the magnetic flux in the air gap $\phi_{g}$ and the fringing flux $\phi_{f}$ :

$$
\phi_{c}=\phi_{g}+\phi_{f} \text {. }
$$

The permeance of the core is

$$
\mathcal{P}_{c}=\frac{1}{\mathcal{R}_{c}}=\frac{\mu_{r c} \mu_{0} A_{c}}{l_{c}} .
$$

The permeance of the air gap is

$$
\mathcal{P}_{g}=\frac{1}{\mathcal{R}_{g}}=\frac{\mu_{0} A_{c}}{l_{g}}
$$

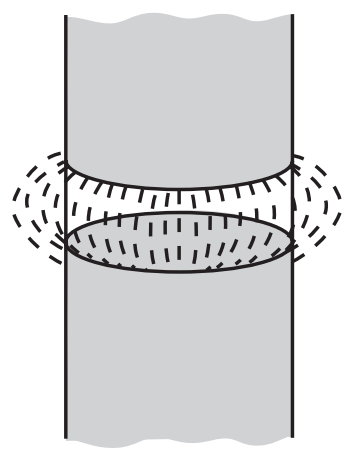

Figure 1.20 Fringing magnetic flux in an air gap.

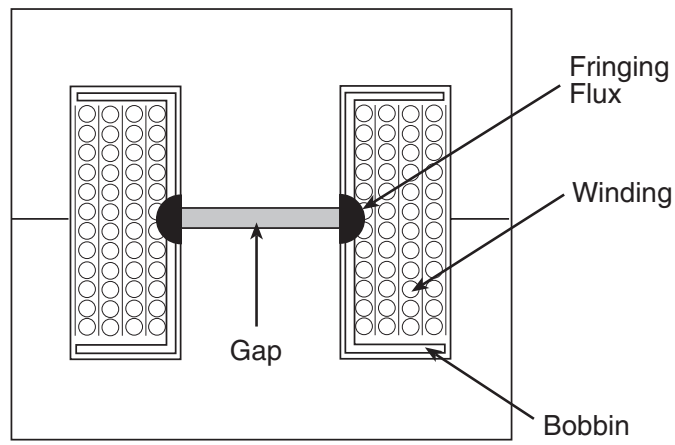

Figure 1.21 Fringing magnetic flux in an inductor with gapped pot core. 


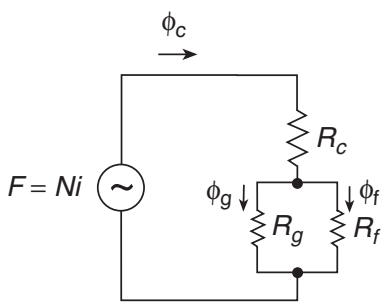

Figure 1.22 Magnetic equivalent circuit of an inductor with an air gap and fringing magnetic flux.

The permeance of the fringing area is

$$
\mathcal{P}_{f}=\frac{1}{\mathcal{R}_{f}}=\frac{\mu_{0} A_{f}}{l_{f}}
$$

where $A_{f}$ is the fringing area and $l_{f}$ is the magnetic path length in the fringing area. Assuming that $A_{g}=A_{c}$, the total reluctance is given by

$$
\begin{aligned}
\mathcal{R} & =\mathcal{R}_{c}+\mathcal{R}_{g} \| \mathcal{R}_{f}=\mathcal{R}_{c}+\frac{\mathcal{R}_{g} \mathcal{R}_{f}}{\mathcal{R}_{g}+\mathcal{R}_{f}}=\frac{\frac{l_{g}}{\mu_{r c} \mu_{0} A_{c}} \times \frac{l_{f}}{\mu_{0} A_{f}}}{\frac{l_{g}}{\mu_{0} A_{g}}+\frac{l_{f}}{\mu_{0} A_{f}}} \\
& =\frac{l_{c}}{\mu_{r c} \mu_{0} A_{c}}+\frac{l_{g} l_{f}}{l_{g} \mu_{0} A_{f}+l_{f} \mu_{0} A_{g}}=\frac{l_{c}}{\mu_{r c} \mu_{0} A_{c}}\left(1+\frac{\mu_{r c} A_{c}}{l_{c}} \frac{l_{g} l_{f}}{l_{f} A_{g}+l_{g} A_{f}}\right) \\
& =\frac{l_{c}}{\mu_{r c} \mu_{0} A_{c}}\left(1+\frac{\mu_{r c} l_{g}}{l_{c}} \frac{1}{1+\frac{l_{g} A_{f}}{l_{f} A_{g}}}\right) .
\end{aligned}
$$

Hence, the inductance of an inductor with an air gap and a fringing flux is given by

$$
L_{f}=\frac{N^{2}}{\mathcal{R}}=N^{2} /\left[\frac{l_{c}}{\mu_{r c} \mu_{0} A_{c}}\left(1+\frac{\mu_{r c} l_{g}}{l_{c}} \frac{1}{1+\frac{l_{g} A_{f}}{l_{f} A_{g}}}\right)\right] .
$$

Neglecting the permeance of the core, the total permeance of the air gap an the fringing area is

$$
\mathcal{P}=\mathcal{P}_{g}+\mathcal{P}_{f}=\frac{\mu_{0} A_{c}}{l_{g}}+\frac{\mu_{0} A_{f}}{l_{f}}=\frac{\mu_{0} A_{c}}{l_{g}}\left(1+\frac{A_{f} l_{g}}{A_{c} l_{f}}\right)=\frac{\mu_{0} A_{c} F_{f}}{l_{g}}=F_{f} \mathcal{P}_{g} .
$$

Thus, $\mathcal{R}=\mathcal{R}_{g} / F_{f}$. The inductance of the inductor with an air gap and the fringing flux is

$$
L_{f}=\mathcal{P} N^{2}=\frac{\mu_{0} A_{c} N^{2}}{l_{g}}+\frac{\mu_{0} A_{f} N^{2}}{l_{f}}=\frac{\mu_{0} A_{c} N^{2}}{l_{g}}\left(1+\frac{A_{f} l_{g}}{A_{c} l_{f}}\right)=\frac{\mu_{0} A_{c} N^{2} F_{f}}{l_{g}}=F_{f} L,
$$

where the fringing factor is defined as the ratio of the inductance with an air gap and with the fringing effect $L_{f}$ to the ideal inductance with an air gap and with no fringing effect,

$$
F_{f}=\frac{L_{f}}{L}=1+\frac{A_{f} l_{g}}{A_{c} l_{f}}
$$

Thus, the fringing effect increases the inductance. The number of turns required to obtain a desired inductance is

$$
N=\sqrt{\frac{l_{g} L}{\mu_{0} A_{c} F_{f}}}
$$


If the air gap is enclosed by the winding, the fringing flux is reduced, lowering the value of $F_{f}$. However, the inductor losses increase by as much as 5 times. To reduce these losses, the winding should be moved away from the air gap by a distance equal to two to three times the air gap length $l_{g}$. Short distributed air gaps significantly reduce the fringing flux and power losses. Cores with a large relative permeability require long air gaps, which increase the fringing flux.

Consider a round core with a single air gap. The cross-sectional area of the core with diameter $D_{c}$ is given by

$$
A_{c}=\frac{\pi}{4} D_{c}^{2} .
$$

It is difficult to determine the cross-sectional area $A_{f}$ and the mean path length $l_{f}$ of the magnetic flux. Assume that the outer diameter of the fringing magnetic flux is $D_{f}=D_{c}+2 l_{g}$ and the mean magnetic path length of the fringing flux is $l_{f}=2 l_{g}$. The cross-sectional area of the fringing flux is

$$
A_{f}=\frac{\pi}{4}\left(D_{c}+2 l_{g}\right)^{2}-\frac{\pi}{4} D_{c}^{2}=\pi l_{g}\left(D_{c}+l_{g}\right) .
$$

Hence, the fringing factor is

$$
F_{f}=1+\frac{A_{f} l_{g}}{A_{c} l_{f}}=1+2 l_{g}\left(\frac{1}{D_{c}}+\frac{l_{g}}{D_{c}^{2}}\right) .
$$

The permeance of the air gap is

$$
\mathcal{P}_{g}=\frac{\mu_{0} A_{c}}{l_{g}}=\frac{\pi \mu_{0} D_{c}^{2}}{4 l_{g}} .
$$

The permeance of the fringing area is

$$
\mathcal{P}_{f}=\frac{\mu_{0} A_{f}}{l_{f}}=\frac{\pi \mu_{0}\left(D_{c}+l_{g}\right)}{2} .
$$

The total permeance of the air gap and the fringing area is

$$
\mathcal{P}=\mathcal{P}_{g}+\mathcal{P}_{f}=\frac{\mu_{0} A_{c}}{l_{g}}+\frac{\mu_{0} A_{f}}{l_{f}}=\frac{\pi \mu_{0} D_{c}^{2}}{4 l_{g}}+\frac{\pi \mu_{0}\left(D_{c}+l_{g}\right)}{2} .
$$

The inductance is

$$
L_{f}=\mathcal{P} N^{2}=\left[\frac{\pi \mu_{0} D_{c}^{2}}{4 l_{g}}+\frac{\pi \mu_{0}\left(D_{c}+l_{g}\right)}{2}\right] N^{2}=\frac{\pi \mu_{0} D_{c}^{2} N^{2}}{4 l_{g}}\left[1+\frac{2 l_{g}\left(D_{c}+l_{g}\right)}{D_{c}^{2}}\right]=F_{f} L,
$$

where

$$
F_{f}=1+\frac{2 l_{g}\left(D_{c}+l_{g}\right)}{D_{c}^{2}} \approx 1+\frac{2 l_{g}}{D_{c}} \text { for } l_{g} \ll D_{c} .
$$

\section{Example 1.6}

An inductor with an air gap has a round core with $D_{c}=10 \mathrm{~mm}$ and $l_{g}=1 \mathrm{~mm}$. Find $F_{f}$ and $N_{f} / N$.

Solution: The fringing factor is

$$
F_{f}=\frac{L_{f}}{L}=1+\frac{2 l_{g}\left(D_{c}+l_{g}\right)}{D_{c}^{2}}=1+\frac{2 \times 1 \times(10+1)}{10^{2}}=1.22 .
$$

The ratio of the turns is

$$
\frac{N_{f}}{N}=\frac{1}{\sqrt{F_{f}}}=\frac{1}{\sqrt{1.22}}=0.9054 .
$$

If we use the approximate equation for $F_{f}$, we have

$$
F_{f}=\frac{L_{f}}{L}=1+\frac{2 l_{g}}{D_{c}}=1+\frac{2 \times 1}{10}=1.2
$$


and

$$
\frac{N_{f}}{N}=\frac{1}{\sqrt{F_{f}}}=\frac{1}{\sqrt{1.2}}=0.9123 .
$$

Consider a core with a single rectangular air gap. The dimensions of the air gap are $a$ and $b$. The cross-sectional area of the air gap is

$$
A_{c}=a b .
$$

Assume that the outer dimensions of the fringing magnetic flux are $A=a+2 l_{g}$ and $B=b+2 l_{g}$, and the mean magnetic path length of the fringing flux is $l_{f}=2 l_{g}$. The cross-sectional area of the fringing flux is

$$
A_{f}=\left(a+2 l_{g}\right)\left(b+2 l_{g}\right)-a b=2 l_{g}(a+b)+4 l_{g} .
$$

The fringing factor is

$$
F_{f}=\frac{L_{f}}{L}=1+\frac{l_{g}\left(a+b+2 l_{g}\right)}{a b} .
$$

\section{Example 1.7}

An inductor with a single rectangular air gap has $a=10 \mathrm{~mm}, b=20 \mathrm{~mm}$, and $l_{g}=1 \mathrm{~mm}$. Find $F_{f}$ and $N_{f} / N$.

Solution: The fringing factor is

$$
F_{f}=\frac{L_{f}}{L}=1+\frac{l_{g}\left(a+b+2 l_{g}\right)}{a b}=1+\frac{1 \times(10+20+2 \times 1)}{10 \times 20}=1.16 .
$$

The ratio of the turns is

$$
\frac{N_{f}}{N}=\frac{1}{\sqrt{F_{f}}}=\frac{1}{\sqrt{1.16}}=0.9285
$$

The fringing factor given in [5], [10] is described by

$$
F_{f}=1+\frac{a l_{g}}{\sqrt{A_{c}}} \ln \left(\frac{2 w}{l_{g}}\right) \approx 1+\frac{l_{g}}{\sqrt{A_{c}}} \ln \left(\frac{2 w}{l_{g}}\right)
$$

where $w$ is the width of the core window, $a$ lies between 0.85 and 0.95 for round cores and between 1 and 1.1 for rectangular cores. Typical values of the fringing factor $F_{f}$ are between 1.1 and 1.4. The fringing flux reduces the total reluctance of the magnetic path $\mathcal{R}$, and therefore it increases the inductance $L$. The inductance is increased due to the fringing effect and is given by

$$
\begin{aligned}
L_{f}=F_{f} L=\left[1+\frac{a l_{g}}{\sqrt{A_{c}}} \ln \left(\frac{2 w}{l_{g}}\right)\right] L & =\left[1+\frac{a l_{g}}{\sqrt{A_{c}}} \ln \left(\frac{2 w}{l_{g}}\right)\right] \frac{\mu_{r c} \mu_{0} A_{c} N^{2}}{l_{c}\left(1+\mu_{r c} l_{g}\right)} \\
& =\frac{\mu_{0} A_{c} N^{2} F_{f}}{l_{g}+\frac{l_{c}}{\mu_{r c}}} .
\end{aligned}
$$

Therefore, the number of turns $N$ to obtain a required inductance $L$ of an inductor with an air gap and the fringing effect should be reduced to

$$
N_{f}=\sqrt{\frac{L_{f}\left(l_{g}+\frac{l_{c}}{\mu_{r c}}\right)}{\mu_{0} A_{c} F_{f}}}=\frac{N}{\sqrt{F_{f}}} .
$$

Fringing flux generates eddy currents, which cause hot spots in both the core and the winding, resulting in power losses. The winding, banding, and clips should be kept away from the fringing flux to reduce power losses. A distributed air gap along the magnetic path reduces winding loss, as compared to the winding loss due to a single air gap. 


\subsubsection{Inductance of Strip Transmission Line}

Consider a strip transmission line, where $d$ is the distance between the conductors, $l$ is the length of the strip, and $w$ is the width of the strip. The magnetic field intensity between conducting parallel plates is

$$
H=\frac{I l}{w},
$$

resulting in the flux linkage

$$
\lambda=\iint_{S} \mathbf{B} \cdot d \mathbf{S}=\int_{0}^{l} \int_{0}^{d} \frac{\mu I}{w} d x d z=\frac{\mu I l d}{w} .
$$

Hence, the inductance of the strip transmission line is given by

$$
L=\frac{\lambda}{I}=\frac{\mu d l}{w} .
$$

\subsubsection{Inductance of Coaxial Cable}

The inductance of a coaxial cable of inner radius $a$, outer radius $b$, and length $l_{w}$ is given by

$$
L=\frac{\mu l_{w}}{2 \pi} \ln \left(\frac{b}{a}\right) .
$$

\subsubsection{Inductance of Two-Wire Transmission Line}

The inductance of a two-wire transmission line of round conductor of radius $a$, distance between the conductor centers $d$, and length $l_{w}$ is

$$
\begin{aligned}
L & =\frac{\mu l_{w}}{\pi} \cosh ^{-1}\left(\frac{d}{2 a}\right)=\frac{\mu l_{w}}{\pi} \ln \left[\frac{d}{2 a}+\sqrt{\left(\frac{d}{2 a}\right)^{2}-1}\right] \\
& \approx \frac{\mu l_{w}}{\pi} \ln \left(\frac{d}{a}\right), \text { for }\left(\frac{d}{2 a}\right)^{2} \gg 1,
\end{aligned}
$$

where $\cosh ^{-1} x \approx \ln (2 x)$ for $x \ll 1$.

\subsection{Inductance Factor}

Equation (1.189) for the inductance can be written as

$$
L=\frac{\mu_{r c} \mu_{0} A_{c} N^{2}}{l_{c}}=A_{L} N^{2} .
$$

The specific inductance of a core, also called the core inductance factor, is defined as the inductance per single turn,

$$
A_{L}=\frac{L}{N^{2}}=\frac{\mu_{r c} \mu_{0} A_{c}}{l_{c}}=\frac{1}{\mathcal{R}}=\mathcal{P}\left(\frac{\mathrm{H}}{t^{2}}\right) .
$$

Each core of different materials, shapes, and sizes will have a unique value of $A_{L}$, some of which are not easy to predict analytically, especially for complex core shapes. Core manufacturers give the values of $A_{L}$ in data sheets. 
The specific inductance (or the inductance index) $A_{L}$ is usually specified in henries per turn, in millihenries per 1000 turns, or in microhenries per 100 turns for cores without and with air gaps. If the specific inductance $A_{L}$ is expressed in henries per turn, the number of turns is given by

$$
N=\sqrt{\frac{L(\mathrm{H})}{A_{L}}} .
$$

If the specific inductance $A_{L(1000)}$ is expressed in millihenries per 1000 turns, the inductance is given by

$$
L=\frac{A_{L(1000)} N^{2}}{(1000)^{2}}(\mathrm{mH})
$$

and the number of turns is

$$
N=1000 \sqrt{\frac{L(\mathrm{mH})}{A_{L(1000)}}} .
$$

For most ferrite cores, the specific inductance $A_{L(100)}$ is specified in microhenries per 100 turns. In this case, the inductance is given by

$$
L=\frac{A_{L(100)} N^{2}}{(100)^{2}}(\mu \mathrm{H}) .
$$

To compute the required number of turns $N$ in order to achieve a desired inductance $L$ in microhenries, the following formula can be used for ferrite cores:

$$
N=100 \sqrt{\frac{L(\mu \mathrm{H})}{A_{L(100)}}} .
$$

Common values of $A_{L(100)}$ are 16, 25, 40, 63, 100, 250, 400, and so on.

Air core inductors are linear devices because the relationship $B=\mu_{0} H$ is linear. In general, inductors with magnetic cores are nonlinear devices as the relationship between $B$ and $H$ is nonlinear. For $B<B_{s}$, inductors can be modeled as linear devices.

\section{Example 1.8}

The relative permeability of the Ferroxcube ferrite magnetic core material is $\mu_{r c}=1800$. The toroidal core made of this material has inner diameter $d=13.1 \mathrm{~mm}$, external diameter $D=23.7 \mathrm{~mm}$, and height $h=7.5 \mathrm{~mm}$. Find the specific inductance of this core. What is the inductance of the inductor with this core if the number of turns is $N=10$ ?

Solution: The magnetic path length is

$$
l_{c}=\pi \frac{d+D}{2}=\pi \frac{13.1+23.7}{2}=57.805 \mathrm{~mm}
$$

and the cross-sectional area of the core is

$$
A_{c}=h \frac{(D-d)}{2}=7.5 \times 10^{-3} \times \frac{(23.7-13.1) \times 10^{-3}}{2}=39.75 \times 10^{-6} \mathrm{~m}^{2} .
$$

Hence, the specific inductance of the core is

$$
A_{L}=\frac{\mu_{r c} \mu_{0} A_{c}}{l_{c}}=\frac{1800 \times 4 \pi \times 10^{-7} \times 39.75 \times 10^{-6}}{57.805 \times 10^{-3}}=1.5554 \mu \mathrm{H} / \text { turn } .
$$

The inductance at $N=10$ is

$$
L=N^{2} A_{L}=10^{2} \times 1.5554 \times 10^{-6}=155.54 \mu \mathrm{H} .
$$




\subsection{Magnetic Energy}

The instantaneous power of an inductor is

$$
p(t)=i_{L}(t) v_{L}(t)=i_{L}\left(L \frac{d i_{L}}{d t}\right)=L i_{L} \frac{d i_{L}}{d t} .
$$

Power is the time rate of change of energy $P=W / \Delta t$. The instantaneous magnetic energy stored in the magnetic field of an inductor without an air gap is given by

$$
\begin{aligned}
w_{m}(t) & =\int_{0}^{t} p(t) d t=\int_{0}^{t} i_{L} v_{L} d t=\int_{0}^{t} i_{L} L \frac{d i_{L}}{d t} d t=L \int_{0}^{i_{L}} i_{L} d i_{L}=\frac{1}{2} L i_{L}^{2}=\frac{1}{2} \lambda i_{L}=\frac{\lambda^{2}}{2 L} \\
& =\frac{1}{2} \frac{N^{2}}{\mathcal{R}} i_{L}^{2}=\frac{1}{2} \frac{N^{2}}{\frac{l_{c}}{\mu_{r c} \mu_{0} A_{c}}}\left(\frac{H l_{c}}{N}\right)^{2}=\frac{1}{2} \mu_{r c} \mu_{0} H^{2} A_{c} l_{c} \\
& =\frac{B^{2} l_{c} A_{c}}{2 \mu_{r c} \mu_{0}}=\frac{B^{2} V_{c}}{2 \mu_{r c} \mu_{0}}(\mathrm{~J})
\end{aligned}
$$

where $V_{c}=l_{c} A_{c}$ is the core volume, $v_{L}=L d i_{L} / d t, i_{L}=\lambda / L, L=N^{2} / \mathcal{R}$, and $H=B / \mu$. The magnetic energy is proportional to the core volume $V_{c}$ and the flux density $B$, and it is inversely proportional to the core relative permeability $\mu_{r c}$.

The magnetic energy density is

$$
w_{m}=\frac{W_{m}}{V_{c}}=\frac{B^{2}}{2 \mu_{r c} \mu_{0}}=\frac{1}{2} \mu_{r c} \mu_{0} H^{2}=\frac{1}{2} \mu H^{2}\left(\frac{\mathrm{J}}{\mathrm{m}^{3}}\right) .
$$

For an inductor with an air gap, the energy stored in the gap is

$$
W_{g}=\frac{B^{2} l_{g} A_{g}}{2 \mu_{0}} \approx \frac{B^{2} l_{g} A_{c}}{2 \mu_{0}}
$$

where $A_{g} \approx A_{c}$. The energy stored in the core is

$$
W_{c}=\frac{B^{2} l_{c} A_{c}}{2 \mu_{r c} \mu_{0}} .
$$

The total energy stored in an inductor with an air gap is equal to the sum of the energy stored in the gap $W_{g}$ and the energy stored in the core $W_{c}$ :

$$
W_{m}=W_{g}+W_{c}=\frac{B^{2} A_{c}}{2 \mu_{0}}\left(l_{g}+\frac{l_{c}}{\mu_{r c}}\right) .
$$

For $l_{g} \gg l_{c} / \mu_{r c}$, almost all the inductor energy is stored in the air gap:

$$
W_{m} \approx W_{g}=\frac{B^{2} l_{g} A_{g}}{2 \mu_{0}} \approx \frac{B^{2} l_{g} A_{c}}{2 \mu_{0}} .
$$

The maximum energy that can be stored in an inductor is limited by the core saturation flux density $B_{s}$, the core volume $V_{c}$, and the core relative permeability $\mu_{r c}$. The maximum energy stored in an inductor with a core without an air gap is given by

$$
W_{c(\max )}=\frac{B_{s}^{2} l_{c} A_{c}}{2 \mu_{r c} \mu_{0}}=\frac{B_{s}^{2} V_{c}}{2 \mu_{r c} \mu_{0}} .
$$

The maximum energy that can be stored in an inductor with a core with an air gap is

$$
W_{g(\max )}=\frac{B_{s}^{2} l_{g} A_{c}}{2 \mu_{0}}=\frac{B_{s}^{2} V_{g}}{2 \mu_{0}}
$$

where $V_{g}=l_{g} A_{c}$ is the air gap volume. The ratio of the two energies is

$$
\frac{W_{g(\max )}}{W_{c(\max )}}=\frac{l_{g}}{l_{c}} \mu_{r c} .
$$


A winding represents a series combination of an inductance and a frequency-dependent resistance. The quality factor of an inductor at a given frequency $f$ is defined as

$$
Q_{L o}=\frac{\omega L}{r_{L}},
$$

where $r_{L}$ is the equivalent series resistance at frequency $f$.

\section{Example 1.9}

A Ferroxcube 528T500-4C4 ferrite magnetic core has $A_{c}=1.17 \mathrm{~cm}^{2}, l_{c}=8.49 \mathrm{~cm}$, and $\mu_{r c}=125$. (a) Determine the maximum magnetic energy that can be stored in the inductor with this core. (b) Determine the maximum magnetic energy that can be stored in the inductor whose core has an air gap $l_{g}=0.5 \mathrm{~mm}$. (c) Find the ratio of the maximum magnetic energies.

Solution: The saturation flux density $B_{s}$ for ferrite cores is $B_{s}=0.3 \mathrm{~T}$ at room temperature. At $T=100^{\circ} \mathrm{C}$, the saturation flux density $B_{s}$ for ferrite cores decreases by a factor of 2 . Thus,

$$
B_{s}=\frac{0.3}{2}=0.15 \mathrm{~T} \text {. }
$$

The maximum magnetic energy that can be stored in the inductor is

$$
W_{m(\max )}=\frac{B_{s}^{2} l_{c} A_{c}}{2 \mu_{r c} \mu_{0}}=\frac{0.15^{2} \times 8.49 \times 10^{-2} \times 1.17 \times 10^{-4}}{2 \times 125 \times 4 \pi \times 10^{-7}}=0.711 \mathrm{~mJ} .
$$

The maximum magnetic energy that can be stored in the inductor whose core contains an air gap is

$$
W_{g(\max )}=\frac{B_{s}^{2} l_{g} A_{c}}{2 \mu_{0}}=\frac{0.15^{2} \times 0.5 \times 10^{-3} \times 1.17 \times 10^{-4}}{2 \times 4 \pi \times 10^{-7}}=0.5237 \mathrm{~mJ} .
$$

Hence,

$$
\frac{W_{g(\max )}}{W_{m(\max )}}=\frac{0.5237}{0.711}=0.7362 .
$$

\subsection{Self-Resonant Frequency}

The distributed capacitance between the winding turns acts like a shunt capacitance, conducting a high-frequency current. This capacitance is called a stray capacitance or a self-capacitance $C_{s}$ [32], [33]. It depends on the winding geometry, the proximity of turns, core, and shield, and the permittivity of the dielectric insulator, in which the winding wire is coated. A single, well-spaced secondary winding is recommended to reduce the interwinding capacitance. The core should be insulated to reduce the capacitance between the winding and the core. The inductance and the self-capacitance form a parallel resonant circuit, having a fundamental (parallel) self-resonant frequency

$$
f_{r}=\frac{1}{2 \pi \sqrt{L C_{s}}} .
$$

Below this frequency, the inductor impedance is inductive. Above the self-resonant frequency $f_{r}$, the inductor impedance is capacitive. Therefore, the useful operating frequency range of an inductor is usually from de to $0.9 f_{r}$. 


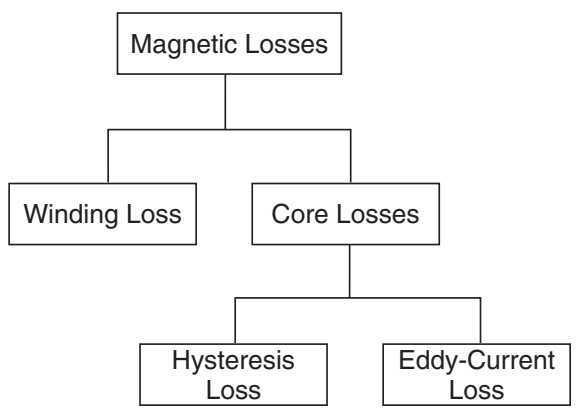

Figure 1.23 Classification of power losses in magnetic components.

\subsection{Classification of Power Losses in Magnetic Components}

Figure 1.23 shows a classification of power losses in magnetic components. These losses can be categorized into winding (or copper) loss $P_{R w}$ and core losses $P_{C}$. In turn, core losses can be divided into hysteresis loss $P_{H}$ and eddy-current loss $P_{E}$ :

$$
P_{C}=P_{H}+P_{E}
$$

Hence, the total inductor power loss $P_{L}$ is given by

$$
P_{L}=P_{R w}+P_{C}=P_{R w}+P_{H}+P_{E} .
$$

There are two kinds of eddy-current losses: the skin-effect loss and the proximity-effect loss. Both these effects cause current crowding. Eddy-current losses are magnetically induced losses.

\subsection{Noninductive Coils}

In some applications, it is desired to have a noninductive coil. Precision resistors are usually noninductive. For example, current probes require noninductive resistors. A noninductive coil is usually made using closely spaced, parallel windings, called the bifilar winding, as illustrated in Figure 1.24(a). Therefore, every coil turn has an adjacent turn, which carries current in the opposite direction. The magnetic fields generated by adjacent turns cancel each other, as shown in Figure 1.24(b). As a result, the coil does not store magnetic flux and presents no inductance.

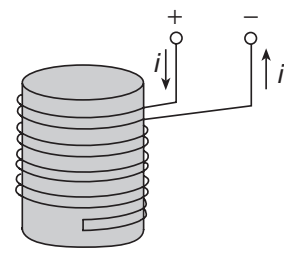

(a)

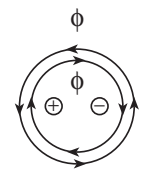

(b)

Figure 1.24 Noninductive coil. (a) Bifilar winding (b) Magnetic flux cancellation. 


\subsection{Summary}

- Magnetic fields can be categorized as self, proximity, and fringing magnetic fields.

- The instantaneous field vector is a function of position and time.

- The phasor field vector is a function of position only.

- The magnetomotive force $\mathcal{F}=N i$ is a source in magnetic circuits.

- A changing current in an inductor produces a changing magnetic flux, which induces voltage between the terminals of the inductor.

- Ampère's law states that the line integral of $\mathbf{H}$ around a closed contour $C$ is equal to the current traversing the surface bounded by the contour.

- Faraday's law states that an ac voltage is induced in a coil, which contains a time-varying magnetic flux, regardless of the source of flux.

- According to Faraday's law, the voltage induced in the inductor is proportional to the number of turns $N$ and the time rate of change of the magnetic flux $\phi$.

- The magnetic flux always takes the path with the highest permeability $\mu$.

- The Poynting vector represents the direction and the density of power flow.

- The self-inductance of a wire-wound inductor depends on its geometry and is proportional to the square of the number of turns $N$.

- The reluctance is directly proportional to the length of the magnetic path $l_{c}$ and inversely proportional to the core cross-sectional area $A_{c}$ through which the magnetic flux $\phi$ flows.

- The inductance is proportional to the ratio of the core cross-sectional area to the magnetic path length $A_{c} / l_{c}$.

- The inductance of an inductor with a ferromagnetic core is $\mu_{r c}$ times higher than that of an air core inductor. An inductor has an ampere-turn maximum value of $\left(N I_{m}\right)_{\max }$ limited by the core saturation flux density $B_{s}$.

- An air gap is used to prevent core saturation and to make the inductance almost independent of $\mu_{r c}$, yielding good inductance repeatability.

- The air gap contains nearly all of the magnetic field energy.

- An air gap in the core increases the energy storage capability of an inductor or a transformer.

- The core relative permeability $\mu_{r c}$ varies considerably with temperature and current. Therefore, it is desirable to maintain $\mathcal{R}_{c} \ll \mathcal{R}_{g}$ to achieve a stable inductance.

- The inductance of an inductor with an air gap is lower than the inductance of an inductor without an air gap.

- Whenever the core is excited, the fringing flux is present around the air gap, increasing the inductance and causing power losses. The fringing field decreases substantially within one air-gap length $l_{g}$ of the edge of the core.

- Fringing flux represents a larger percentage of the total flux for larger gaps.

- Fringing flux and inductor losses can be reduced by dividing a large air gap into several shorter air gaps.

- The fringing flux reduces the total reluctance $\mathcal{R}$ and increases the inductance $L$. Therefore, the number of turns should be reduced if the exact value of the inductance is required.

- The winding should be moved away from the air gap by a distance of twice the air gap length. 
- The thickness of the shield foils should be low compared to the skin depth. As the distance between the shields decreases, the inductance also decreases.

- The self-resonant frequency of an inductor is the resonant frequency of the resonant circuit formed by the inductance and stray capacitance.

- Power losses in inductors and transformers consist of winding and core losses.

- Core losses consist of hysteresis loss and eddy-current loss.

- The turns should be evenly spaced to achieve consistent inductance and reduce leakage inductance.

\subsection{References}

[1] P. J. Dowell, Effects of eddy currents in transformer winding. Proceedings of the IEE, vol. 113, no. 8, pp. 1387-1394, August 1966.

[2] J. Jongsma, High-frequency ferrite power transformer and choke design, Part 3: Transformer winding design; Part 4: Improved method of power choke design. Philips Electronic Components and Materials, Technical Publication, no. 27, Philips, The Netherlands, 1986.

[3] A. Kennelly, F. Laws, and P. Pierce, Experimental research on skin effect in conductors. Transactions of the AIEE, vol. 34, pp. 1953-2021, 1915.

[4] G. F. Partridge, Philosophical Magazine, 7th Series, vol. 22, July-December 1943, p. 675.

[5] R. Lee, L. Wilson, and C. E. Carter, Electronic Transformers and Circuits, 3rd edn. New York: John Wiley \& Sons, Inc. 1988.

[6] J. Lammeraner and M. Stafl, Eddy Currents. Cleveland, OH: CRS Press, 1966.

[7] J. Ebert, Four terminal parameters of HF inductor. Bulletin de l'Académie Polonaise des Sciences, Série des Sciences Techniques, no. 5, 1968.

[8] E. C. Snelling, Soft Ferrites: Properties and Applications. London: Iliffe Books, 1969.

[9] R. L. Stall, The Analysis of Eddy Currents. Oxford: Clarendon Press, 1974, pp. 21-27.

[10] W. T. McLyman, Transformer and Inductor Design Handbook, 3rd edn. New York: Marcel Dekker, 2004.

[11] J. K. Watson, Applications of Magnetism. New York: John Wiley \& Sons, Inc., 1980.

[12] E. Bennet and S. C. Larsen, Effective resistance of alternating currents of multilayer windings. Transactions of the AIEE, vol. 59, pp. 1010-1017, 1940.

[13] R. L. Perry, Multiple layer series connected winding design for minimum losses. IEEE Transactions on Power Apparatus and Systems, vol. PAS-98, pp. 116-123, January/February 1979.

[14] B. Carsten, High frequency conductor losses in switch mode magnetics. Proceedings of PCI, Munich, Germany, 1986, pp. 161-182.

[15] J. P. Vandalec and P. D. Ziogos, A novel approach for minimizing high-frequency transformer copper loss. IEEE Transactions on Power Electronics, vol. 3, pp. 266-276, July 1988.

[16] A. M. Urling, V. A. Niemela, G. R. Skutt, and T. G. Wilson, Characterizing high frequency effects in transformer windings: A guide to several significant papers. IEEE Transactions on Power Electronics Specialists Conference, 1989, pp. 373-385.

[17] J. A. Ferreira, Electromagnetic Modelling of Power Electronic Converters. Boston: Kluwer Academic, 1989.

[18] J. G. Kassakian, M. F. Schlecht, and G. C. Verghese, Principles of Power Electronics. Reading, MA: Addison-Wesley, 1991.

[19] M. Bartoli, A. Reatti, and M. K. Kazimierczuk, High-frequency models of ferrite inductors. Proceedings of the IEEE International Conference on Industrial Electronics, Controls, Instrumentation, and Automation (IECON'94), Bologna, Italy, September 5-9, 1994, pp. 1670-1675.

[20] M. Bartoli, A. Reatti, and M. K. Kazimierczuk, Predicting the high-frequency ferrite-core inductor performance. Proceedings of the Conference of Electrical Manufacturing and Coil Winding, Chicago (Rosemont), IL, September 27-29, 1994, pp. 409-413.

[21] M. Bartoli, A. Reatti, and M. K. Kazimierczuk, Modeling iron-powder inductors at high-frequencies. Proceedings of the IEEE Industry Applications Society Annual Meeting, Denver, CO, October 2-7, 1994, pp. $1225-1232$.

[22] J. A. Ferreira, Improved analytical modeling of conductive losses in magnetic components. IEEE Transactions on Power Electronics, vol. 9, pp. 127-131, January 1994.

[23] M. Bartoli, N. Noferi, A. Reatti, and M. K. Kazimierczuk, Modeling winding losses in high-frequency power inductors. Journal of Circuits, Systems and Computers, vol. 5, no. 3, pp. 65-80, March 1995. 
[24] N. Mohan, T. M. Undeland, and W. P. Robbins, Power Electronics, 3rd edn. Hoboken, NJ: John Wiley \& Sons, Inc., 2003.

[25] M. Bartoli, N. Nefari, A. Reatti, and M. K. Kazimierczuk, Modeling litz-wire winding losses in high-frequency power inductors. Proceedings of the IEEE Power Electronics Specialists Conference, Baveno, Italy, June 24-27, 1996, pp. 1690-1696.

[26] R. Petkov, Optimum design of a high-power high-frequency transformer. IEEE Transactions on Power Electronics, vol. 11, no. 1, pp. 33-42, January 1996.

[27] Chee-Mun Ong, Dynamic Simulation of Electric Machinery. Upper Saddle River, NJ: Prentice Hall PTR, 1998, pp. 38-40, 45-46, and 87-90.

[28] G. Bertotti, Hysteresis of Magnetism. San Diego, CA: Academic Press, 1998.

[29] W. G. Hurley, W. H. Wolfe, and J. G. Breslin, Optimized transformer design: Inclusive of high-frequency effects. IEEE Transactions on Power Electronics, vol. 13, no. 4, pp. 651-659, July 1998.

[30] W. G. Hurley, E. Gath, and J. G. Breslin, Optimizing the ac resistance of multilayer transformer windings with arbitrary current waveforms. IEEE Transactions on Power Electronics, vol. 15, no. 2, pp. 369-376, March 2000.

[31] M. K. Kazimierczuk, G. Sancineto, U. Reggiani, and A. Massarini, Small-signal high-frequency model of ferrite inductors. IEEE Transactions on Magnetics, vol. 35, pp. 4185-4191, September 1999.

[32] U. Reggiani, G. Sancineto, and M. K. Kazimierczuk, High-frequency behavior of laminated iron-core inductors for filter applications. Proceedings of the IEEE Applied Power Electronics Conference, New Orleans, February 6-10, 2000, pp. 654-660.

[33] G. Grandi, M. K. Kazimierczuk, A. Massarini, U. Reggiani, and G. Sancineto, Model of laminated iron-core inductors. IEEE Transactions on Magnetics, vol. 40, no. 4, pp. 1839-1845, July 2004.

[34] K. Howard and M. K. Kazimierczuk, Eddy-current power loss in laminated power cores. Proceedings of the IEEE International Symposium on Circuits and Systems, Sydney, Australia, May 7-9, 2000, paper III-668, pp. $668-672$.

[35] A. Reatti and M. K. Kazimierczuk, Comparison of various methods for calculating the ac resistance of inductors. IEEE Transactions on Magnetics, vol. 37, pp. 1512-1518, May 2002.

[36] G. Grandi, M. K. Kazimierczuk, A. Massarini, and U. Reggiani, Stray capacitance of single-layer solenoid air-core inductors. IEEE Transactions on Industry Applications, vol. 35, no. 5, pp. 1162-1168, September 1999.

[37] A. Massarini and M. K. Kazimierczuk, Self-capacitance of inductors. IEEE Transactions on Power Electronics, vol. 12, no. 4, pp. 671-676, July 1997.

[38] H. A. Wheeler, Simple inductance formulas for radio coils. Proceedings of the IRE, vol. 16, no. 10, pp. 1398-1400, October 1928.

[39] R. W. Erickson and D. Maksimović, Fundamentals of Power Electronics. Norwell, MA: Kluwer Academic, 2001.

[40] A. Van den Bossche and V. C. Valchev, Inductors and Transformers for Power Electronics. Boca Raton, FL: Taylor \& Francis, 2005.

[41] F. E. Terman, Radio Engineers' Handbook. New York: McGraw-Hill, 1943.

[42] J. C. Maxwell, A Treatise of Electricity and Magnetism, 3rd edn. New York: Dover Publishing, 1997.

[43] N. Das and M. K. Kazimierczuk, An overview of technical challenges in the design of current transformers. Electrical Manufacturing Conference, Indianapolis, October 24-26, 2005.

[44] T. Suetsugu and M. K. Kazimierczuk, Integration of Class DE inverter for dc-dc converter on-chip power supplies. IEEE International Symposium on Circuits and Systems, Kos, Greece, May 21-24, 2006, pp. 3133-3136.

[45] M. K. Kazimierczuk, Pulse-Width Modulated DC-DC Power Converters. Chichester: John Wiley \& Sons, Ltd, 2008.

[46] M. K. Kazimierczuk, RF Power Amplifiers, Chichester: John Wiley \& Sons, Ltd, 2008.

[47] T. Suetsugu and M. K. Kazimierczuk, Integration of Class DE synchronized dc-dc converter on-chip power supplies. IEEE Power Electronics Specialists Conference, Jeju, South Korea, June 21-24, 2006.

[48] www.mag-inc.com, www.ferroxcube.com, www.ferrite.de, www.micrometals.com, and www.metglas.com.

\subsection{Review Questions}

1.1. What is the magnetomotive force?

1.2. What is the magnetic field intensity?

1.3. What is the magnetic flux density? 
1.4. What is the magnetic linkage?

1.5. Define magnetic susceptibility.

1.6. Define relative permeability.

1.7. What is the reluctance of an inductor?

1.8. What is the magnetic circuit? Give an example.

1.9. Can magnetic field exist in a good conductor?

1.10. State Ampère's circuital law.

1.11. State Faraday's law.

1.12. State Lenz's law.

1.13. What is Joule's law?

1.14. What is the point form of Ohm's law?

1.15. Give Maxwell's equations.

1.16. Give Maxwell's equations for good conductors.

1.17. What is core saturation?

1.18. Define the inductance of a linear inductor.

1.19. Define the inductance of a nonlinear inductor.

1.20. Give an expression for the current of a nonlinear inductor.

1.21. What is the core inductance factor?

1.22. How is the inductance of a coil related to its number of turns?

1.23. What is the effect of an air gap on the inductance?

1.24. What is the fringing factor?

1.25. What is the effect of an air gap on core saturation?

1.26. Where is the magnetic energy stored in an inductor with an air gap?

1.27. Is the magnetic field intensity in the air gap higher or lower than that in the core?

1.28. Is the magnetic flux density in the air gap higher or lower than that in the core?

1.29. What is the volt-second balance?

1.30. Give expressions for magnetic energy in terms of $H$ and $B$.

1.31. What are the mechanisms of power losses in magnetic components?

1.32. What are winding losses?

1.33. What is hysteresis loss?

1.34. What is eddy-current loss?

1.35. What are the effects of eddy currents on winding conductors and magnetic cores?

1.36. What is the self-resonant frequency?

1.37. What is the difference between fringing flux and leakage flux?

1.38. The line integral of the magnetic field intensity $H$ over a closed contour is zero. What is the net current flowing through the surface enclosed by the contour? 


\subsection{Problems}

1.1. A current flows in the inner conductor of a long coaxial cable and returns through the outer conductor. What is the magnetic field intensity in the region outside the coaxial cable? Explain why.

1.2. Sketch the shape of the magnetic field around a current-carrying conductor and show how the direction of the field is related to the direction of the current in the conductor.

1.3. A toroidal inductor has $N=20$ turns, inner radius $a=1 \mathrm{~cm}$, outer radius $b=2 \mathrm{~cm}$, and height $h=1 \mathrm{~cm}$. The core relative permeability is $\mu_{r}=100$. Find the inductance.

1.4. An inductor has $N=300$ turns, current $I=0.1 \mathrm{~A}$, and $B=0.5 \mathrm{~T}$. The cross-sectional area $A_{c}=4 \mathrm{~cm}^{2}$, and the length $l_{c}=15 \mathrm{~cm}$. Find the magnetic field intensity, the magnetic flux, and the flux linkage.

1.5. An inductor has $\mu_{r}=800, N=700, \phi=0.4 \mathrm{mWb}, l_{w}=22 \mathrm{~cm}$, and $A_{c}=4 \times 10^{-4} \mathrm{~m}^{2}$. Find the current $I$.

1.6. An inductor has $L=100 \mu \mathrm{H}, l_{c}=2.5 \mathrm{~cm}$, and $A_{c}=2 \mathrm{~cm}^{2}$. Find the number of turns $N$ for (a) $\mu_{r c}=1$, (b) $\mu_{r c}=25$ and no air gap, (c) $\mu_{r c}=25$ and $l_{g}=3 \mathrm{~mm}$, and (d) $\mu_{r c}=2500$ and $l_{g}=3 \mathrm{~mm}$.

1.7. A core has $A_{L}=30 \mu \mathrm{H} / 100$ turns. Find $N$ to make an inductor of $L=1 \mu \mathrm{H}$.

1.8. A toroidal core inductor has: $N=500, \mu_{r}=200, A_{c}=4 \mathrm{~cm}^{2}, r=2 \mathrm{~cm}, I_{m}=1 \mathrm{~A}, f=$ $10 \mathrm{MHz}, \rho_{w}=\rho_{C u}=1.724 \times 10^{-6} \Omega \mathrm{cm}$, and $\rho_{c}=10^{5} \Omega \mathrm{m}$. Find $L, A_{L}, \mathcal{R}, H_{m}, B_{m}, \phi_{m}$, and $\lambda_{m}$.

1.9. A toroidal core of $\mu_{r c}=3000$ has a mean radius $R=80 \mathrm{~mm}$ and a circular cross section with radius $b=25 \mathrm{~mm}$. The core has an air gap $l_{g}=3 \mathrm{~mm}$ and a current $I$ flows in a 500-turn winding to produce a magnetic flux of $10^{-4} \mathrm{~Wb}$. Neglect the leakage flux. (a) Determine the reluctances of the air gap and the core. (b) Find $B_{g}$ and $H_{g}$ in the air gap and $B_{c}$ and $H_{c}$ in the core. (c) Find the required current $I$.

1.10. An inductor has $N=100, A_{c}=1 \mathrm{~cm}^{2}, B_{s}=0.3 \mathrm{~T}$, and $v_{L}=V_{L m} \cos \omega t=10 \cos \omega t$ (V). Find $\lambda(t)$ and $f_{\min }$.

1.11. Derive an expression for the internal and external inductances of a two-wire transmission line consisting of two parallel round conductors of radius $a$, which carry currents $I$ in opposite directions. The axis-to-axis distance between the two conductors is $d \gg a$. 